\title{
A DONSKER THEOREM TO SIMULATE ONE-DIMENSIONAL PROCESSES WITH MEASURABLE COEFFICIENTS*
}

\author{
Pierre Étoré ${ }^{1}$ And Antoine Lejay ${ }^{2}$
}

\begin{abstract}
In this paper, we prove a Donsker theorem for one-dimensional processes generated by an operator with measurable coefficients. We construct a random walk on any grid on the state space, using the transition probabilities of the approximated process, and the conditional average times it spends on each cell of the grid. Indeed we can compute these quantities by solving some suitable elliptic PDE problems.
\end{abstract}

Mathematics Subject Classification. 60J60, 65C.

Received May 29, 2006.

\section{INTRODUCTION}

In this paper we provide a scheme for simulating one-dimensional processes generated by Divergence Form Operators (DFO), with measurable coefficients. Those are operators of type

$$
L=\frac{\rho}{2} \nabla(a \nabla \cdot)+b \nabla \cdot .
$$

A sufficient condition for $L$ to generate a continuous Markov process is that $a$ and $\rho$ are uniformly elliptic and bounded and that $b$ is bounded. Note that DFOs contain the case of non divergence form operators.

A lot of probabilistic numerical methods allow us already to treat the case of smooth coefficients $a, \rho$ and $b$ (see [12] for example). However we wish to deal with irregular coefficients. Indeed DFOs appear in the modelization of diffusion phenomena and the irregularity of the coefficients can reflect the irregularity of the media the particle is evolving in. This is interesting in a wide variety of physical situations, for example in fluid mechanics in porous media [19], in the modelization of the brain [9], and can also be used in finance [5,6].

In [23], Stroock and Zheng have used the theory of Dirichlet forms to give some results on the approximation of diffusions by Markov chains. These results are valid in the multidimensional case and with discontinuous coefficients, but are very general and cannot be used to build some numerical scheme in a simple way.

Keywords and phrases. Monte Carlo methods, Donsker theorem, one-dimensional process, scale function, divergence form operators, Feynman-Kac formula, elliptic PDE problem.

* This research was supported by the French Groupe de Recherche MOMAS.

1 CERMICS, École Nationale des Ponts et Chaussées, 6 et 8 avenue Blaise Pascal Cité Descartes, Champs sur Marne, 77455 Marne la Vallée Cedex 2, France; Pierre.Etore@cermics.enpc.fr

2 Projet OMEGA, Institut Élie Cartan (UMR 7502, Nancy-Université, CNRS, INRIA) and INRIA Lorraine, Campus scientifique, BP 239, 54506 Vandoeuvre-lès-Nancy Cedex, France; Antoine.Lejay@iecn.u-nancy.fr 
Recently some authors have provided schemes in the case of coefficients having some discontinuities, in dimension $d=1$. The case of dimension $d>1$ appears to be a challenging problem.

In [16] (see also [17]) M. Martinez has treated the case $\rho=1$ and $a$ and $b$ having one point of discontinuity in some $x_{0}$. He uses the fact that the process $X$ generated by $L$ solves the following Stochastic Differential Equation with Local Time (SDELT),

$$
X_{t}=X_{0}+\int_{0}^{t} \sqrt{a \rho}\left(X_{s}\right) \mathrm{d} W_{s}+\int_{0}^{t}\left[\frac{\rho a^{\prime}}{2}+b\right]\left(X_{s}\right) \mathrm{d} s+\sum_{x \in \mathcal{I}} \frac{a(x+)-a(x-)}{a(x+)+a(x-)} L_{t}^{x}(X),
$$

where $\mathcal{I}$ is the set of the points of discontinuity of $a$. Using a proper space transformation he gets rid of the local time in (2) and then uses an Euler scheme. To estimate the speed of convergence of his method, he needs $a$ to be $\mathrm{C}^{6}$ outside the point of discontinuity. The initial condition has to be $\mathrm{C}^{4}$ almost everywhere and to satisfy other restrictive conditions.

In [15] (see also [16]) Lejay and Martinez propose a different scheme. First they perform a piecewise constant approximation of the coefficients. Second they use another space transformation, to obtain a process that behaves locally like a Skew Brownian Motion (SBM) which is known to solve

$$
Y_{t}=Y_{0}+W_{t}+\beta L_{t}^{y}(Y)
$$

with $\beta \in[-1,1]$. Third they use an exact simulation method of the Skew Brownian Motion (SBM), based on the simulation of the exit times of a Brownian motion. In general the whole algorithm is slow and costly but allows us to treat the case of coefficients $a, \rho$ and $b$ being right continuous with left limits (r.c.l.l.) and of class $\mathrm{C}^{1}$ except on countable set of points, without cluster point. Besides the initial condition can be taken in $\mathrm{H}^{1}$, and the algorithm is well adapted to the case of coefficients being flat on large intervals outside their points of discontinuity.

In $[7,8]$, one of the authors has used the same kind of space transformation to obtain a process $Y=\Phi(X)$ that behaves like a SBM around each point of a regular grid $\Phi(\mathfrak{g})=\{k h \mid k \in \mathbb{Z}\}$. He then performs an asymmetric random walk on $\Phi(\mathfrak{g})$ to approach the transformed process $Y$. The random walk is asymmetric because its transition probabilities can differ from $1 / 2$, but the time spent by the approximating process on each cell is always $h^{2}$. This corresponds to the average time spent by $Y$ on each cell of $\Phi(\mathfrak{g})$. The obtained algorithm is easy to implement, fast, and allows us to treat the same cases as in [15] except that the initial condition has to be taken in $\mathrm{W}^{1, \infty} \cap \mathrm{H}^{1} \cap \mathrm{C}_{0}$. Notice that the convergence rate is of order $\mathcal{O}\left(h^{1 / 2}\right)$, which is not surprising comparing to Donsker's theorem.

One of the limitations of this latter algorithm is that, in order to get a regular grid $\Phi(\mathfrak{g})$ for $Y=\Phi(X)$, one has to construct a grid $\mathfrak{g}$ for $X$ that depends on the coefficients $a, \rho$ and $b$. Besides one has to assume that the coefficients are of class $\mathrm{C}^{1}$ except on a countable set of points without cluster point. Indeed one deeply relies on the theory of SDELT which requires this assumption.

Here we do not use SDELTs and propose a more general algorithm that allows us to get rid of both these limitations. We can take measurable coefficients and any grid on the real line. Roughly the idea is the following:

Let $\mathfrak{g}=\left\{x_{j}\right\}_{j \in J}$ be a set of points on the real line, with $x_{j}<x_{j+1}, \forall j \in J$. Let $X$ be the process generated by $L$ defined by (1).

At each point $x_{j}$ in $\mathfrak{g}$, knowing $X$ is in $x_{j}$, we ask ourselves two questions:

(1) What is the probability $\pi(j, j+1)$ that the next point of $\mathfrak{g}$ the process $X$ reaches is $x_{j+1}$ and not $x_{j-1}$ ?

(2) Knowing $X$ has gone to $x_{j+1}$ (respectively to $x_{j-1}$ ) what is the average time $T(j,+)$ (respectively $T(j,-))$ it has taken to go from $x_{j}$ to $x_{j+1}$ (respectively from $x_{j}$ to $x_{j-1}$ )?

We will show (Sects. 4 and 5 ) that it is possible to link the quantities $T(j,+)$ and $T(j,-)$ with the solutions of some elliptic problems involving the operator $L$.

Suppose we know all the $\pi(j, j+1)$ 's, $T(j,+)$ 's and $T(j,-)$ 's and we want an approximation of $X$ at time $t$. We set $\hat{t}=0$ and perform the path on $\mathfrak{g}$ of a random walk $S^{\mathfrak{g}}$ with transition probabilities the $\pi(j, j+1)$ 's. 

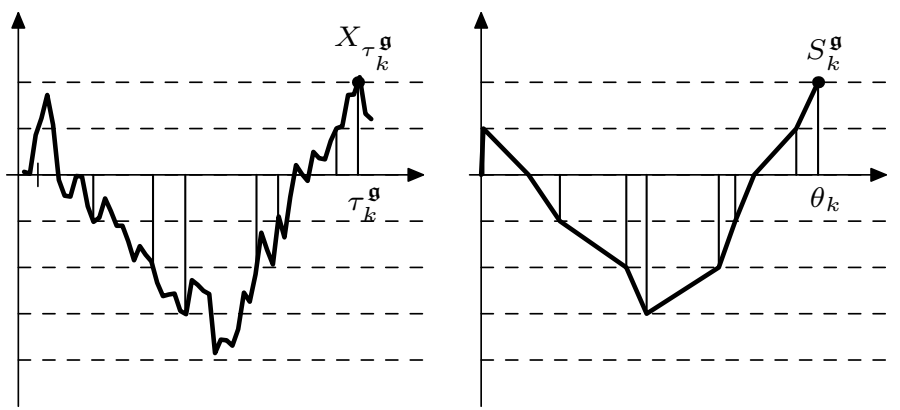

FIGURE 1. One path of $X_{t}$ and the linear interpolation of the corresponding path of $S_{K^{\mathfrak{g}}(t)}^{\mathfrak{g}}$.

Each time $S^{\mathfrak{g}}$ passes from one point of $\mathfrak{g}$ to another one we add to $\hat{t}$ the corresponding $T(j, \pm)$. When $\hat{t}$ is greater than $t$ we stop the algorithm and return the current position of $S^{\mathfrak{g}}$.

One can think of this construction as a generalization of the Donsker theorem. Assume the minimum and the maximum step size of the grid $\mathfrak{g}$ is related to some parameter $h$. One construct a random walk $\left(S_{k}^{\mathfrak{g}}\right)_{k}$ and an integer value function $K^{\mathfrak{g}}(t)$, such that $S^{\mathfrak{g}}$ reflects the successive positions of the trajectories of $X$ on $\mathfrak{g}$, and $S_{K{ }^{\mathfrak{g}(t)}}^{\mathfrak{g}}$ converges in distribution to $X_{t}$ for each $t \geq 0$, as $h$ tends to zero.

If $\tau_{k}^{\mathfrak{g}}$ denotes the first time after $\tau_{k-1}^{\mathfrak{g}}$ at which $X_{t}$ passes through a point of $\mathfrak{g}$ different from $X_{\tau_{k-1}^{\mathfrak{g}}}$, and $\theta_{k}$ is the time constructed at which we consider $S_{k}^{\mathfrak{g}}$, then the two-dimensional path obtained by joining the $\left(\theta_{k}, S_{k}^{h}\right)$ 's by segments is an approximation of the path obtained by joining the $\left(\tau_{k}^{\mathfrak{g}}, X_{\tau_{k}}\right)$ 's by segments (see Fig. 1).

If $a=\rho=1$ and $b=0$, then $X$ is a Brownian motion and if $\mathfrak{g}$ is the uniform grid with step $h$, then $\left(S_{k}^{\mathfrak{g}}\right)_{k}$ is a simple random walk, $\theta_{k+1}-\theta_{k}=h^{2}$, and the convergence result we prove implies the Donsker theorem.

The scheme is very easy to implement if we first perform some suitable piecewise constant approximations of the coefficients. By doing this it is possible to assume that the grid is constructed at a mesoscopic scale, and that the piecewise constant coefficients correspond to some effective coefficients of highly oscillating coefficients at a small scale, as the homogenization theory proves it $[1,11]$. Numerical tests show a good behaviour of this algorithm.

Besides, compared to the previous ones, this scheme seems more adaptable to the multidimensional case. Indeed it relies strongly on the link between PDE theory and the one of Markov processes, and involves elliptic PDEs that could still be written in dimension 2 or 3 .

Some hypotheses and notations. We will denote by $G$ the state space of the processes we will consider, which is here a connected interval of $\mathbb{R}$. The set $G$ will be also the definition domain of the coefficients of the DFOs, and of the functions they can be applied.

For $u$ in $\mathrm{L}^{2}(G)$ we denote by $\frac{\mathrm{d} u}{\mathrm{~d} x}$ the derivative of $u$ in the distributions' sense. We classically denote by $\mathrm{H}^{1}(G)$ the space of functions in $\mathrm{L}^{2}(G)$ such that $\frac{\mathrm{d} u}{\mathrm{~d} x} \in \mathrm{L}^{2}(G)$, and by $\mathrm{H}_{0}^{1}(G)$ the closure of $\mathrm{C}_{\mathrm{c}}^{\infty}(G)$ in $\mathrm{H}^{1}(G)$ equipped with the norm $\left(\|u\|_{2}^{2}+\left\|\frac{\mathrm{d} u}{\mathrm{~d} x}\right\|_{2}^{2}\right)^{1 / 2}$.

Outline of the paper. In Section 2 we recall some basic facts on DFOs and the associated processes. In Section 3 we present the convergence results we will need. In Section 4 we link the conditional moments of exit times we will need with the solutions of some suitable elliptic PDE problems. In Section 5 we present our scheme. In Section 6 we show this scheme converges and evaluate its rate of convergence. Section 7 deals with numerical considerations. 


\section{DFO AND Markov PROCESSES}

In this section we recall some facts on processes generated by a DFO. As these results are standard we do not much emphasize on the proofs.

\subsection{The existence of a Markov process}

For $0<\lambda<\Lambda<\infty$ let us denote by $\mathfrak{E} \mathfrak{r l}(\lambda, \Lambda)$ the set of functions $f$ on $G$ that are measurable and such that

$$
\forall x \in G, \quad \lambda \leq f(x) \leq \Lambda
$$

We denote by $\mathfrak{B}(\Lambda)$ the set of functions $f$ on $G$ that are measurable and such that

$$
\forall x \in G, \quad|f(x)| \leq \Lambda
$$

For $a, \rho \in \mathfrak{E} \mathfrak{l l}(\lambda, \Lambda)$ and $b \in \mathfrak{B}(\Lambda)$, we will denote by $\mathfrak{L}(a, \rho, b)$ the DFO defined by

$$
L=\frac{\rho}{2} \frac{\mathrm{d}}{\mathrm{d} x}\left(a \frac{\mathrm{d}}{\mathrm{d} x}\right)+b \frac{\mathrm{d}}{\mathrm{d} x}
$$

and

$$
D(L)= \begin{cases}\left\{f \in \mathrm{H}_{0}^{1}(G),\right. & \left.a \frac{\mathrm{d} f}{\mathrm{~d} x} \in \mathrm{H}^{1}(G)\right\} \text { if } G \text { is finite and for Dirichlet b.c., } \\ \left\{f \in \mathrm{H}^{1}(G), a \frac{\mathrm{d} f}{\mathrm{~d} x} \in \mathrm{H}^{1}(G)\right\} \text { if } G \text { is infinite or for Neumann b.c. }\end{cases}
$$

For any measure $g(x) \mathrm{d} x$ with a bounded density $g$ we denote by $\mathrm{L}^{2}(G, g)$ the set of the functions in $\mathrm{L}^{2}(G)$ endowed with the scalar product

$$
(f, h) \longmapsto \int_{G} f(x) h(x) g(x) \mathrm{d} x .
$$

Note that when we simply write $\mathrm{L}^{2}(G)$ the involved measure is implicitly the Lebesgue one. With these notations we have the following theorem.

Theorem 2.1. Consider $a, \rho \in \mathfrak{E} \mathfrak{l l}(\lambda, \Lambda)$ and $b \in \mathfrak{B}(\Lambda)$. Fix $T>0$.

i) The operator $\mathfrak{L}(a, \rho, b)$ is the infinitesimal generator of a strongly continuous semigroup of contraction $\left(S_{t}\right)_{0 \leq t \leq T}$ on $\mathrm{L}^{2}\left(G, \rho^{-1}\right)$.

ii) Moreover $\left(S_{t}\right)_{0 \leq t \leq T}$ is a Feller semigroup. Thus $\mathfrak{L}(a, \rho, b)$ is the infinitesimal generator of a Markov process $\left(X_{t}, 0 \leq t \leq T\right)$.

iii) The process $\left(X_{t}, 0 \leq t \leq T\right)$ has continuous trajectories.

Proof. See [13], Chapter 0.

Sometimes it is more convenient to have an operator of the form $\mathfrak{L}(a, \rho, 0)$ than one of the form $\mathfrak{L}(a, \rho, b)$ (see Sect. 2.2). It is possible to pass from one form to the other.

Proposition 2.2. Consider $a, \rho \in \mathfrak{E} \mathfrak{l l}(\lambda, \Lambda)$ and $b \in \mathfrak{B}(\Lambda)$. Set $(L, D(L))=\mathfrak{L}(a, \rho, b)$ and choose $[\ell, r] \subset G$. For $x_{0} \in[\ell, r]$, let $\Psi$ be defined by

$$
\Psi(x)=2 \int_{x_{0}}^{x} \frac{b(y)}{a(y) \rho(y)} \mathrm{d} y, \forall x \in[\ell, r] .
$$

Consider on $[\ell, r]$ the operator $(\bar{L}, D(\bar{L}))=\mathfrak{L}\left(a \mathrm{e}^{\psi}, \rho \mathrm{e}^{-\Psi}, 0\right)$. The restriction on $[\ell, r]$ of $(L, D(L))$ is equal to $(\bar{L}, D(\bar{L}))$. 
Proof. As $b$ is in $\mathfrak{B}(\Lambda)$ it is possible to show that $a \mathrm{e}^{\Psi}$ and $\rho \mathrm{e}^{-\Psi}$ are in $\mathfrak{E} \mathfrak{r}\left(\lambda^{\prime}, \Lambda^{\prime}\right)$ on $[\ell, r]$, with $\lambda^{\prime}$ and $\Lambda^{\prime}$ depending on $\lambda, \Lambda$ and $\ell-r$.

As the functions $\mathrm{e}^{ \pm \Psi}$ are bounded and have bounded classical derivatives they are in $\mathrm{H}^{1}([\ell, r])$. So the properties of the product of functions of class $\mathrm{H}^{1}$ (see [4] Cor. VIII.9) allow us to assert that, on $[\ell, r], D(L)=$ $D(\bar{L})$ and that

This concludes the proof.

$$
\forall f \in D(L), \frac{\rho \mathrm{e}^{-\Psi}}{2} \frac{\mathrm{d}}{\mathrm{d} x}\left(a \mathrm{e}^{\Psi} \frac{\mathrm{d} f}{\mathrm{~d} x}\right)=\frac{\rho}{2} \frac{\mathrm{d}}{\mathrm{d} x}\left(a \frac{\mathrm{d} f}{\mathrm{~d} x}\right)+b \frac{\mathrm{d} f}{\mathrm{~d} x} .
$$

\subsection{Scale function and speed measure}

We recall here the definitions of the scale function and the speed measure of a process $X$ on an interval $G$ of the real line. We follow [3], Chapter 16.

Definition 2.3. The scale function of a Markov process $X$ on $G$ is the strictly increasing function $s$, unique up to a linear transformation, such that for all $[\ell, r] \subset G$, and for all $x \in[\ell, r]$,

$$
\mathbb{P}^{x}\left[X_{\tau}=r\right]=\frac{s(x)-s(\ell)}{s(r)-s(\ell)}
$$

where $\tau=\inf \left\{t \geq 0 \mid X_{t} \in\{\ell, r\}\right\}$.

The scale function of the Brownian motion is the identity function. A process $X$ is said to be on its natural scale if its scale function is the identity function. When $X$ is not on its natural scale it is easy to check that $s(X)$ is.

Definition 2.4. Let $X$ be a Markov process on $G$ that is on its natural scale. The speed measure of $X$ is the unique measure $m$ on $G$ such that, for all $[\ell, r] \subset G$, and for all $x \in[\ell, r]$, with $\tau=\inf \left\{t \geq 0 \mid X_{t} \in\{\ell, r\}\right\}$, we have

where,

$$
\mathbb{E}^{x}[\tau]=\int_{\ell}^{r} G_{[\ell, r]}(x, z) m(\mathrm{~d} z),
$$

$$
G_{[\ell, r]}(x, z)= \begin{cases}\frac{2(x-\ell)(r-z)}{r-\ell}, & \forall x, z \in[\ell, r], x \leq z, \\ \frac{2(z-\ell)(r-x)}{r-\ell}, & \forall x, z \in[\ell, r], x \geq z \\ 0, & \text { otherwise. }\end{cases}
$$

When $X$ is not on its natural scale, there is still a notion of speed measure (see Problem 16.7 in [3]).

Lemma 2.5. Let $X$ be a Markov process on $G$ and $s$ its scale function. Assume the speed measure of $s(X)$ is $V(x) \mathrm{d} x$. We have for all $[\ell, r] \subset G$ and for all $x \in[\ell, r]$, with $\tau=\inf \left\{t \geq 0 \mid X_{t} \in\{\ell, r\}\right\}$,

$$
\mathbb{E}^{x}[\tau]=\int_{\ell}^{r} G_{[\ell, r]}(x, y) V(s(y)) s^{\prime}(y) \mathrm{d} y
$$

where,

$$
G_{[\ell, r]}(x, y)= \begin{cases}\frac{2(s(x)-s(\ell))(s(r)-s(y))}{s(r)-s(\ell)}, & \forall x, y \in[\ell, r], x \leq y, \\ \frac{2(s(y)-s(\ell))(s(r)-s(x))}{s(r)-s(\ell)}, & \forall x, y \in[\ell, r], x \geq y, \\ 0, & \text { otherwise. }\end{cases}
$$

We call $m(\mathrm{~d} x)=V(s(x)) s^{\prime}(x) \mathrm{d} x$ the speed measure of $X$. 
Proof. We have $\mathbb{E}^{x}[\tau]=\mathbb{E}^{s(x)}[\tilde{\tau}]$, with $\tilde{\tau}=\inf \left\{t \geq 0 \mid s\left(X_{t}\right) \in\{s(\ell), s(r)\}\right\}$. As $s(X)$ is on its natural scale it suffices to use in (3) the change of variable $z=s(y)$ to complete the proof.

In dimension one to give the scale function and the speed measure of a Markov process suffices to fully characterize it.

Given a Markov process generated by a DFO we can directly determine its scale function and speed measure.

Proposition 2.6. Consider a, $\rho \in \mathfrak{E} \mathfrak{E r}(\lambda, \Lambda)$. Let $X$ be generated by $\mathfrak{L}(a, \rho, 0)$. Fix $x_{0} \in G$. The scale function and speed measure of $X$ are given by

$$
\begin{aligned}
s(x) & =\int_{x_{0}}^{x} \frac{\mathrm{d} y}{a(y)}, \\
\text { and } m(\mathrm{~d} x) & =\frac{\mathrm{d} x}{\rho(x)} .
\end{aligned}
$$

Proof. See [13] Chapter 5, where it is made use of convergence results proved in [10].

Remark 2.7. Note that as the scale function $s$ is unique up to a linear transform, the choice of the reference point $x_{0}$ in (5) is arbitrary. We may choose any point in $G$.

\subsection{Using estimates on the kernel}

We will need the following lemma, proved by Stroock in [22].

Lemma 2.8. Consider $a, \rho \in \mathfrak{E} \mathfrak{r}(\lambda, \Lambda)$ and $b \in \mathfrak{B}(\Lambda)$. Choose $T>0$. Let $X$ be generated by $\mathfrak{L}(a, \rho, b)$. There exists a constant $A$ depending on $\lambda, \Lambda$ and $T$, such that, for all $(t, x) \in[0, T] \times G$ and any $d>0$,

$$
\mathbb{P}^{x}\left[\sup _{s \in[0, t]}\left|X_{s}-x\right| \geq d\right] \leq A \exp \left(-\frac{A d^{2}}{t}\right) .
$$

Proof. In [22] the lemma is proved for $\rho=1$ and $b=0$. However the proof is based on Aronson's estimates that remain valid with any $b \in \mathfrak{B}(\Lambda)$ provided that we are at some finite horizon $T$. Besides Aronson's estimates concern the transition probability density of $X$ with respect to the Lebesgue measure in the case $\rho=1$. In the case $\rho \neq 1$, we have the same estimates for the density with respect ot the measure $\rho^{-1}(x) \mathrm{d} x$. As $\rho^{-1}$ is in $\mathfrak{E} \mathfrak{r r}(1 / \Lambda, 1 / \lambda)$, we finally get the same result.

\section{Convergence Results}

We first assert very general convergence results concerning DFOs. They lie on the theory of $G$-convergence, about which we can refer the reader to [24] and [25]. We first assert a result concerning elliptic problems.

Theorem 3.1. Assume $G$ is finite. Consider $a \in \mathfrak{E} \mathfrak{l}(\lambda, \Lambda)$ and $b \in \mathfrak{B}(\Lambda)$. Let $\left(a^{n}\right)$ be a sequence of $\mathfrak{E} \mathfrak{l r}(\lambda, \Lambda)$ and $\left(b^{n}\right)$ a sequence of $\mathfrak{B}(\Lambda)$ such that

$$
\frac{1}{a^{n}} \frac{\mathrm{L}^{2}(G)}{n \rightarrow \infty} \frac{1}{a} \text { and } \frac{b^{n}}{a^{n}} \frac{\mathrm{L}^{2}(G)}{n \rightarrow \infty} \frac{b}{a} .
$$

Set $(L, D(L))=\mathfrak{L}(a, 1, b)$ and for each integer $n,\left(L^{n}, D\left(L^{n}\right)\right)=\mathfrak{L}\left(a^{n}, 1, b^{n}\right)$. Let $\left(f^{n}\right)$ be a sequence converging in $\mathrm{H}^{-1}$ to some $f$. Let $u$ be the solution to the problem Lu $=-f$ submitted to uniform Dirichlet boundary conditions, and for each $n$ let $u^{n}$ be the solution to $L^{n} u^{n}=-f^{n}$.

Then the sequence $\left(u^{n}\right)$ converges weakly in $\mathrm{H}_{0}^{1}(G)$ to $u$.

Proof. This a direct consequence of Theorems 5 and 17 in [24]. 
Remark 3.2. If $G$ is infinite or we want to treat Neumann b.c. the conclusion of Theorem 3.1 still holds, with a weak convergence in $\mathrm{H}^{1}(G)$, but under the assumption the weak convergences $(6)$ hold in $\mathrm{L}^{2}(G)_{\text {loc }}$.

In [25] the authors applied the $G$-convergence theory to parabolic problems. In [21] the author used this to prove some results on the convergence in law of processes generated by DFOs.

Theorem 3.3. Consider $a, \rho \in \mathfrak{E} \mathfrak{l l}(\lambda, \Lambda)$ and $b \in \mathfrak{B}(\Lambda)$. Let $\left(a^{n}\right)$ and $\left(\rho^{n}\right)$ two sequences of $\mathfrak{E} \mathfrak{l l}(\lambda, \Lambda)$ and $\left(b^{n}\right)$ a sequence of $\mathfrak{B}(\Lambda)$ such that

$$
\frac{1}{a^{n}} \frac{\mathrm{L}^{2}(G)_{\mathrm{loc}}}{n \rightarrow \infty} \frac{1}{a}, \frac{1}{\rho^{n}} \frac{\mathrm{L}^{2}(G)_{\mathrm{loc}}}{n \rightarrow \infty} \frac{1}{\rho} \text { and } \frac{b^{n}}{a^{n} \rho^{n}} \frac{\mathrm{L}^{2}(G)_{\mathrm{loc}}}{n \rightarrow \infty} \frac{b}{a \rho}
$$

Let us denote by $S$ and $X$ respectively the semigroup and the process generated by $\mathfrak{L}(a, \rho, b)$ and by $\left(S^{n}\right)$ and $\left(X^{n}\right)$ the sequences of semigroups and processes generated by the sequence of operators $\mathfrak{L}\left(a^{n}, \rho^{n}, b^{n}\right)$.

Then for any $T>0$ and any $f \in \mathrm{L}^{2}(G)$,

i) The function $S_{t}^{n} f(x)$ converges weakly in $\mathrm{L}^{2}(0, T ; \mathrm{E})$ to $S_{t} f(x)$, where $\mathrm{E}=\mathrm{H}_{0}^{1}(G)$ in the case of Dirichlet b.c. and $\mathrm{E}=\mathrm{H}^{1}(G)$ in the case of an infinite $G$ or for Neumann b.c.

ii) The continuous version of $S_{t}^{n} f(x)$ converges uniformly on each compact of $(0, T) \times G$ to the continuous version of $S_{t} f(x)$.

iii) $\left(X_{t}^{n}, t \geq 0\right) \underset{n \rightarrow \infty}{\longrightarrow}\left(X_{t}, t \geq 0\right)$ in distribution.

Proof. See [25] and [21]. See also [15].

We will finally need the following result concerning the convergence of the exit times of a family of processes.

Proposition 3.4. Choose $[\ell, r] \subset G$. Consider $\left(X^{n}\right)$ that converges in law to $X$. Define $\tau=\inf \left\{t \geq 0 \mid X_{t} \in\right.$ $\{\ell, r\}\}$ and for each $n, \tau^{n}=\inf \left\{t \geq 0 \mid X_{t}^{n} \in\{\ell, r\}\right\}$. Then for all $T>0$ and for any starting point $x$,

$$
\mathbb{P}^{x} \circ\left(X^{n}, \tau^{n}\right)^{-1} \underset{n \rightarrow \infty}{\longrightarrow} \mathbb{P}^{x} \circ(X, \tau)^{-1}
$$

with respect to the topology of $\mathrm{C}([0, T], \mathbb{R}) \times \mathbb{R}$. In particular $X_{. \wedge \tau^{n}}^{n}$ converges in law to $X_{. \wedge \tau}$ under each $\mathbb{P}^{x}$.

Proof. See in [15] the end of the proof of Proposition 3. See also [13] Chapter 0, Section 6. These proofs use the fact that any starting point $x$ is regular for $X$.

\section{Conditional moments of EXit times as SOlutions of Elliptic PROBlems}

To build our algorithm and prove its convergence we will need values and estimates of conditional moments of exit times of the approximated process $X$ from an interval $[\ell, r] \subset G$. In order to get them, we link in this section such conditional moments with the solutions of some suitable elliptic problems.

When no special mention is made, we mean by the solution $u$ of an elliptic PDE involving an operator $(L, D(L))$, the continuous version of its solution. Indeed that is necessary if we want to identify $u(x)$ with the expectation starting from $x$ of some functional of the process generated by $(L, D(L))$.

In the sequel the intervals $[\ell, r]$ will be the cells of the grid on $G$ we use to build our algorithm. As the size of these cells will tend to zero, we make in this section the reasonable assumption that

$$
r-\ell \leq 2
$$

We mainly want to prove the following proposition.

Proposition 4.1. Consider $a, \rho \in \mathfrak{E} \mathfrak{l l}(\lambda, \Lambda)$ and $b \in \mathfrak{B}(\Lambda)$. Define the DFO $(L, D(L))$ by $\mathfrak{L}(a, \rho, b)$. Let $X$ be the Markov process generated by $(L, D(L))$. Choose $[\ell, r] \subset G$ and define $\tau=\inf \left\{t \geq 0 \mid X_{t} \in\{\ell, r\}\right\}$. For all 
$x \in[\ell, r]$ we have that $\mathbb{E}^{x}\left[\tau \mathbb{1}_{\left\{X_{\tau}=r\right\}}\right]=v_{1}(x)$, where $v_{1}$ is solution of

$$
\mathcal{P}_{1}([\ell, r],+) \quad \begin{cases}L v_{1}=-v_{0} & \text { in }(\ell, r), \\ v_{1}=0 & \text { on }\{\ell, r\}\end{cases}
$$

where $v_{0}$ is the solution of

$$
\mathcal{P}_{0}([\ell, r],+)\left\{\begin{array}{l}
L v_{0}=0 \text { in }(\ell, r), \\
v_{0}(\ell)=0, \\
v_{0}(r)=1 .
\end{array}\right.
$$

In the same manner $\mathbb{E}^{x}\left[\tau \mathbb{1}_{\left\{X_{\tau}=\ell\right\}}\right]=v_{1}(x)$, where $v_{1}$ is the solution of $\mathcal{P}_{1}([\ell, r],-)$, which is written as $\mathcal{P}_{1}([\ell, r],+)$ but with $v_{0}$ the solution of

$$
\mathcal{P}_{0}([\ell, r],-) \quad\left\{\begin{array}{l}
L v_{0}=0 \text { in }(\ell, r), \\
v_{0}(\ell)=1 \\
v_{0}(r)=0
\end{array}\right.
$$

To prove Proposition 4.1 we need a few lemmas.

Lemma 4.2. For $a, \rho \in \mathfrak{E V r}(\lambda, \Lambda)$ and $b \in \mathfrak{B}(\Lambda)$, set $(L, D(L))=\mathfrak{L}(a, \rho, b)$. The solution of $\mathcal{P}_{0}([\ell, r],+)$ is given by

$$
v_{0}(x)=\frac{\int_{\ell}^{x} \mathrm{e}^{-\Psi(y)} / a(y) \mathrm{d} y}{\int_{\ell}^{r} \mathrm{e}^{-\Psi(y)} / a(y) \mathrm{d} y}
$$

Proof. Straightforward: this function solves $\mathcal{P}_{0}([\ell, r],+)$ which has a unique solution.

Remark 4.3. The function $v_{0}$ is the scale function of the process generated by $\mathfrak{L}(a, \rho, b)$ (see Props. 2.2 and 2.6). In fact the scale function is build by solving such elliptic problems as $\mathcal{P}_{0}([\ell, r],+)$.

Lemma 4.4. Consider $a$ and $\rho$ in $\mathfrak{E r r}(\lambda, \Lambda) \cap \mathrm{C}^{\infty}(G)$ and $b$ in $\mathfrak{B}(\Lambda) \cap \mathrm{C}^{\infty}(G)$. Set $(L, D(L))=\mathfrak{L}(a, \rho, b)$. Let $X$ be the Markov process generated by $(L, D(L))$. Choose $[\ell, r] \subset G$ and set $\tau=\inf \left\{t \geq 0 \mid X_{t} \in\{\ell, r\}\right\}$. For all $x \in[\ell, r]$ we have $\mathbb{E}^{x}\left[\tau \mathbb{1}_{\left\{X_{\tau}=r\right\}}\right]=v_{1}(x)$, where $v_{1}$ is solution of $\mathcal{P}_{1}([\ell, r],+)$ as defined in Proposition 4.1 .

Proof. First notice $\tau$ has moments of any order (see in [8] proof of Lem. 3.1.2 for some justification).

As $a, \rho$ and $b$ are $\mathrm{C}^{\infty}, L$ can be written

$$
L=\frac{\rho a}{2} \frac{\mathrm{d}^{2}}{\mathrm{~d} x^{2}}+\left[\frac{\rho a^{\prime}}{2}+b\right] \frac{\mathrm{d}}{\mathrm{d} x}
$$

Thus $X$ solves the SDE

$$
\mathrm{d} X_{t}=\sqrt{a \rho}\left(X_{t}\right) \mathrm{d} W_{t}+\left[\frac{\rho a^{\prime}}{2}+b\right]\left(X_{t}\right) \mathrm{d} t
$$

As $a, \rho$ and $b$ are $\mathrm{C}^{\infty}$, the solution $v_{0}$ of $\mathcal{P}_{0}([\ell, r],+)$ as described in Proposition 4.1 is of class $\mathcal{C}^{\infty}$ on $[\ell, r]$ (see Lem. 4.2).

We first notice that because of the boundary conditions in $\mathcal{P}_{0}([\ell, r],+)$ we have $\tau v_{0}\left(X_{\tau}\right)=\tau \mathbb{1}_{\left\{X_{\tau}=r\right\}}$.

Second, for $0 \leq t \leq \tau$, applying the Itô rule to $t v_{0}\left(X_{t}\right)$ and since $L v_{0}=0$, we get

$$
\begin{aligned}
t v_{0}\left(X_{t}\right) & =\int_{0}^{t} v_{0}\left(X_{s}\right) \mathrm{d} s+M_{t}+\int_{0}^{t} s L v_{0}\left(X_{s}\right) \mathrm{d} s \\
& =\int_{0}^{t} v_{0}\left(X_{s}\right) \mathrm{d} s+M_{t}
\end{aligned}
$$


where $M_{t}=\int_{0}^{t \wedge \tau} s v_{0}^{\prime}\left(X_{s}\right) \sqrt{a \rho}\left(X_{s}\right) \mathrm{d} W_{s}$ is a local martingale. As $v_{0}^{\prime}$ is bounded on $[\ell, r]$ it can be shown that $\mathbb{E}\langle M\rangle_{\infty}<\infty$, and thus $M$ is a true martingale. So, taking the expectation,

$$
\mathbb{E}^{x}\left[\tau v_{0}\left(X_{\tau}\right)\right]=\mathbb{E}^{x}\left[\int_{0}^{\tau} v_{0}\left(X_{s}\right) \mathrm{d} s\right] .
$$

Third, because of the smoothness of the coefficients, the solution $v_{1}$ of $\mathcal{P}_{1}([\ell, r],+)$ is $\mathrm{C}^{2}$ on $(\ell, r)$ and continuous on $[\ell, r]$. So using the Feynman-Kac formula we get

$$
\mathbb{E}^{x}\left[\tau \mathbb{1}_{\left\{X_{\tau}=r\right\}}\right]=\mathbb{E}^{x}\left[\int_{0}^{\tau} v_{0}\left(X_{s}\right) \mathrm{d} s\right]=v_{1}(x) \forall x \in[\ell, r] .
$$

This conclude the proof.

Lemma 4.5. Consider $a$ and $\rho$ in $\mathfrak{E} \mathfrak{r r}(\lambda, \Lambda) \cap \mathrm{C}^{\infty}(G)$ and $b$ in $\mathfrak{B}(\Lambda) \cap \mathcal{C}^{\infty}(G)$. Set $(L, D(L))=\mathfrak{L}(a, \rho, b)$. Let $X$ be the Markov process generated by $(L, D(L))$. Choose $[\ell, r] \subset G$ and set $\tau=\inf \left\{t \geq 0 \mid X_{t} \in\{\ell, r\}\right\}$. For all $x \in[\ell, r]$ we have $\mathbb{E}^{x}\left[\tau^{2} \mathbb{1}_{\left\{X_{\tau}=r\right\}}\right]=v_{2}(x)$, where $v_{2}$ is solution of

$$
\mathcal{P}_{2}([\ell, r],+) \quad \begin{cases}L v_{2}=-2 v_{1} & \text { in }(\ell, r), \\ v_{2}=0 & \text { on }\{\ell, r\},\end{cases}
$$

where $v_{1}$ is the solution of $\mathcal{P}_{1}([\ell, r],+)$.

In the same manner $\mathbb{E}^{x}\left[\tau^{2} \mathbb{1}_{\left\{X_{\tau}=\ell\right\}}\right]=v_{2}(x)$, where $v_{2}$ is the solution of $\mathcal{P}_{2}([\ell, r],-)$, which is written as $\mathcal{P}_{2}([\ell, r],+)$ but with $v_{1}$ the solution of $\mathcal{P}_{1}([\ell, r],-)$.

Proof. We treat the case of $\mathbb{E}^{x}\left[\tau^{2} \mathbb{1}_{\left\{X_{\tau}=r\right\}}\right]$ and consider the problems $\mathcal{P}_{i}([\ell, r],+)$ for $i=0,1,2$.

Because $a, \rho$ and $b$ of class $\mathrm{C}^{\infty}$ the functions $v_{0}, v_{1}$ and $v_{2}$ are of class $\mathrm{C}^{2}$ on $(\ell, r)$ and continuous on $[\ell, r]$.

We first notice that we have $\tau^{2} \mathbb{1}_{\left\{X_{\tau}=r\right\}}=\tau^{2} v_{0}\left(X_{\tau}\right)$.

Second for all $0 \leq t<\tau$, the Itô rule applied to $t^{2} v_{0}\left(X_{t}\right)$ leads to

$$
\begin{aligned}
t^{2} v_{0}\left(X_{t}\right) & =2 \int_{0}^{t} s v_{0}\left(X_{s}\right) \mathrm{d} s+M_{t}+\int_{0}^{t} s^{2} L v_{0}\left(X_{s}\right) \mathrm{d} s \\
& =-2 \int_{0}^{t} s L v_{1}\left(X_{s}\right) \mathrm{d} s+M_{t}
\end{aligned}
$$

with $M$ a local martingale with bracket $\langle M\rangle_{t}=\int_{0}^{t \wedge \tau} s^{4}\left[\left(v_{0}^{\prime}\right)^{2} a \rho\right]\left(X_{s}\right) \mathrm{d} s$.

Third, applying the Itô rule to $t v_{1}\left(X_{t}\right)$ for all $0 \leq t<\tau$,

$$
t v_{1}\left(X_{t}\right)=\int_{0}^{t} v_{1}\left(X_{s}\right) \mathrm{d} s+N_{t}+\int_{0}^{t} s L v_{1}\left(X_{s}\right) \mathrm{d} s
$$

with $N$ another local martingale with bracket $\langle N\rangle_{t}=\int_{0}^{t \wedge \tau} s^{2}\left[\left(v_{1}^{\prime}\right)^{2} a \rho\right]\left(X_{s}\right) \mathrm{d} s$. Taking in account (9) we have for all $0 \leq t<\tau$,

$$
t^{2} v_{0}\left(X_{t}\right)=M_{t}+2\left(\int_{0}^{t} v_{1}\left(X_{s}\right) \mathrm{d} s+N_{t}-t v_{1}\left(X_{t}\right)\right) .
$$

As $v_{0}^{\prime}$ and $v_{1}^{\prime}$ are bounded on $[\ell, r]$ it can be shown like in the proof of Lemma 4.4 that $M$ and $N$ are true martingales. So taking the expectation we have

$$
\mathbb{E}^{x}\left[t^{2} v_{0}\left(X_{t}\right)\right]=\mathbb{E}^{x}\left[\int_{0}^{t} 2 v_{1}\left(X_{s}\right) \mathrm{d} s\right]-\mathbb{E}^{x}\left[t v_{1}\left(X_{t}\right)\right]
$$


Making $t$ tend to $\tau$ and using the boundary conditions in $\mathcal{P}_{1}([\ell, r],+)$ we have

$$
\mathbb{E}^{x}\left[\tau^{2} \mathbb{1}_{\left\{X_{\tau}=r\right\}}\right]=\mathbb{E}^{x}\left[\tau^{2} v_{0}\left(X_{\tau}\right)\right]=\mathbb{E}^{x}\left[\int_{0}^{\tau} 2 v_{1}\left(X_{s}\right) \mathrm{d} s\right] .
$$

Using again the Feynman-Kac formula, we finally get that

$$
\mathbb{E}^{x}\left[\tau^{2} \mathbb{1}_{\left\{X_{\tau}=r\right\}}\right]=\mathbb{E}^{x}\left[\int_{0}^{\tau} 2 v_{1}\left(X_{s}\right) \mathrm{d} s\right]=v_{2}(x), \forall x \in[\ell, r],
$$

which ends the proof.

Lemma 4.6. Let $a$ and $\rho$ be in $\mathfrak{E r r}(\lambda, \Lambda) \cap \mathrm{C}^{\infty}(G)$ and $b$ in $\mathfrak{B}(\Lambda) \cap \mathcal{C}^{\infty}(G)$. Set $(L, D(L))=\mathfrak{L}(a, \rho, b)$ and choose $[\ell, r] \subset G$. For all function $f$ continuous and bounded, the solution of

$$
(\mathcal{P}) \begin{cases}L u=-f & \text { in }(\ell, r), \\ u=0 & \text { on }\{\ell, r\},\end{cases}
$$

verifies $\|u\|_{\infty} \leq C\|f\|_{\infty}(r-\ell)^{2}$, where $C$ is a constant depending only on $\lambda$ and $\Lambda$.

Proof. Note first that $(L, D(L))=\mathfrak{L}\left(a \mathrm{e}^{\Psi}, \rho \mathrm{e}^{-\Psi}, 0\right)$ with $\Psi$ defined by $\Psi(x)=2 \int_{\ell}^{x} \frac{b(y)}{a(y) \rho(y)} \mathrm{d} y$.

Let $f$ be continuous and bounded and $u$ the solution of $(\mathcal{P})$. It is of class $\mathrm{C}^{2}$ and by Feynman-Kac we have $u(x)=\mathbb{E}^{x}\left[\int_{0}^{\tau} f\left(X_{s}\right) \mathrm{d} s\right]$. But according to Problem 10 in Section 16.6 in [3] and Proposition 2.6 we have that $\mathbb{E}^{x}\left[\int_{0}^{\tau} f\left(X_{s}\right) \mathrm{d} s\right]=\int_{\ell}^{r} G_{[\ell, r]}(x, y) f(y) \exp (\Psi(y)) \rho^{-1}(y) \mathrm{d} y$, where $G_{[\ell, r]}$ is defined by (4) with $s(x)=$ $\int_{\ell}^{x} \exp (-\Psi(y)) / a(y) \mathrm{d} y$. Thus,

$$
u(x)=\int_{\ell}^{r} G_{[\ell, r]}(x, y) f(y) \exp (\Psi(y)) \rho^{-1}(y) \mathrm{d} y,
$$

and we have an explicit expression of $G_{[\ell, r]}$. After some easy computations and using condition (8) we get

$$
\forall x \in[\ell, r], \exp ( \pm \Psi(x)) \leq \kappa
$$

where $\kappa=\max \left(1, \mathrm{e}^{4 \Lambda / \lambda^{2}}\right)$ und thus,

$$
\forall x, y \in[\ell, r] G_{[\ell, r]} \leq 2 \frac{\kappa}{\lambda}(r-\ell) \text { and } \forall y \in[\ell, r], \exp (\Psi(y)) \rho^{-1}(y) \leq \frac{\kappa}{\lambda} .
$$

Using this in (10) completes the proof.

Lemma 4.7. Let $a$ and $\rho$ be in $\mathfrak{E r r}(\lambda, \Lambda) \cap \mathrm{C}^{\infty}(G)$ and $b$ in $\mathfrak{B}(\Lambda) \cap \mathrm{C}^{\infty}(G)$. Set $(L, D(L))=\mathfrak{L}(a, \rho, b)$. Choose $[\ell, r] \subset G$. Let $v_{1}$ and $v_{2}$ be respectively the solutions of $\mathcal{P}_{1}([\ell, r],+)$ and $\mathcal{P}_{2}([\ell, r],+)$ involving $(L, D(L))$. The following estimates hold

$$
\begin{aligned}
&\left\|v_{1}\right\|_{\infty} \leq C(r-\ell)^{2} \\
&\left\|v_{2}\right\|_{\infty} \leq K(r-\ell)^{4},
\end{aligned}
$$

where $C$ and $K$ are positive constants depending only on $\lambda$ and $\Lambda$.

Proof. We have $\left\|v_{0}\right\|_{\infty} \leq 1$. So (12) follows from Lemma 4.6. Then (13) follows from (12) and the same lemma. 
Proof of Proposition 4.1. We prove the link between $\mathbb{E}^{x}\left[\tau \mathbb{1}_{\left\{X_{\tau}=r\right\}}\right]$ and the solution of $\mathcal{P}_{1}([\ell, r],+)$. The link between $\mathbb{E}^{x}\left[\tau \mathbb{1}_{\left\{X_{\tau}=\ell\right\}}\right]$ and $\mathcal{P}_{1}([\ell, r],-)$ is proved in the same manner.

Step 1. We build two sequences $\left(a^{k}\right)$ and $\left(\rho^{k}\right)$ in $\mathfrak{E} \mathfrak{r l}(\lambda, \Lambda) \cap \mathrm{C}^{\infty}(G)$, and a sequence $\left(b^{k}\right)$ in $\mathfrak{B} \cap \mathrm{C}^{\infty}(G)$, such that

$$
a^{k} \underset{k \rightarrow \infty}{\longrightarrow} a, \rho^{k} \underset{k \rightarrow \infty}{\longrightarrow} \rho \text { and } b^{k} \underset{k \rightarrow \infty}{\longrightarrow} \text { ba.e. }
$$

Set $L^{k}:=\mathfrak{L}\left(a^{k}, \rho^{k}, b^{k}\right)$. Recall that $(L, D(L))=\mathfrak{L}\left(a \mathrm{e}^{\Psi}, \rho \mathrm{e}^{-\Psi}, 0\right)$ and $\left(L^{k}, D\left(L^{k}\right)\right)=\mathfrak{L}\left(a^{k} \mathrm{e}^{\Psi^{k}}, \rho^{k} \mathrm{e}^{-\Psi^{k}}, 0\right)$ with $\Psi(x)=2 \int_{\ell}^{x} \frac{b(y)}{a(y) \rho(y)} \mathrm{d} y$ and $\Psi^{k}(x)=2 \int_{\ell}^{x} \frac{b^{k}(y)}{a^{k}(y) \rho^{k}(y)} \mathrm{d} y$.

We call $v_{0}^{k}$ the solution of the problem $\mathcal{P}_{0}^{k}(x,+)$ which is written like $\mathcal{P}_{0}([\ell, r],+)$ but with $L$ replaced by $L^{k}$. We call $v_{1}^{k}$ the solution of the problem $\mathcal{P}_{1}^{k}([\ell, r],+)$ which is written like $\mathcal{P}_{1}([\ell, r],+)$ but with $L$ replaced by $L^{k}$ and $v_{0}$ replaced by $v_{0}^{k}$. For each $k \in \mathbb{N}$ let $X^{k}$ be the process generated by $\left(L^{k}, D\left(L^{k}\right)\right)$ and $\tau^{k}=\inf \left\{t \geq 0 \mid X_{t}^{k} \in\{\ell, r\}\right\}$.

For each $k \in \mathbb{N}$ we can apply Lemma 4.4 and get that

$$
\mathbb{E}^{x}\left[\tau^{k} \mathbb{1}_{\left\{X_{\tau^{k}}^{k}=r\right\}}\right]=v_{1}^{k}(x), \forall x \in[\ell, r]
$$

Step 2. We show that $v_{1}^{k}(x) \rightarrow v_{1}(x)$ as $k \rightarrow \infty$, for all $x \in[\ell, r]$, where the $v_{1}^{k}$ 's and $v_{1}$ are continuous versions of the solutions of the involved PDEs. We introduce the notations $\hat{a}^{k}=a^{k} \mathrm{e}^{\Psi^{k}}, \hat{\rho}^{k}=\rho^{k} \mathrm{e}^{-\Psi^{k}}, \hat{a}=a \mathrm{e}^{\Psi}$ and $\hat{\rho}=\rho \mathrm{e}^{-\Psi}$.

The generalized solutions $v_{1}^{k}$ and $v_{1}$ are elements of $\mathrm{H}_{0}^{1}((\ell, r))$ that respectively verify,

$$
\left(\frac{1}{2} \frac{\mathrm{d}}{\mathrm{d} x}\left(\hat{a}^{k} \frac{\mathrm{d}}{\mathrm{d} x}\right)\right) v_{1}^{k}=-\frac{v_{0}^{k}}{\hat{\rho}^{k}} \text { and }\left(\frac{1}{2} \frac{\mathrm{d}}{\mathrm{d} x}\left(\hat{a} \frac{\mathrm{d}}{\mathrm{d} x}\right)\right) v_{1}=-\frac{v_{0}}{\hat{\rho}}
$$

By Lemma 4.2,

$$
v_{0}^{k}(x)=\frac{\int_{\ell}^{x} \mathrm{e}^{-\Psi^{k}(y)} / a^{k}(y) \mathrm{d} y}{\int_{\ell}^{r} \mathrm{e}^{-\Psi^{k}(y)} / a^{k}(y) \mathrm{d} y} \text { and } v_{0}(x)=\frac{\int_{\ell}^{x} \mathrm{e}^{-\Psi(y)} / a(y) \mathrm{d} y}{\int_{\ell}^{r} \mathrm{e}^{-\Psi(y)} / a(y) \mathrm{d} y} .
$$

Thus, by dominated convergence, we first get that $v_{0}^{k}$ converges pointwise to $v_{0}$. Again by dominated convergence we can get that $v_{0}^{k} / \hat{\rho}^{k}$ tends to $v_{0} / \hat{\rho}$ in $\mathrm{L}^{2}((\ell, r))$, and thus in $\mathrm{H}^{-1}((\ell, r))$. Note that

$$
\forall k \in \mathbb{N},\left\|v_{0}^{k}\right\|_{\infty} \leq 1 \text { and }\left\|\frac{v_{0}^{k}}{\hat{\rho}^{k}}\right\|_{\infty} \leq \frac{1}{\lambda^{\prime}}
$$

where $\lambda^{\prime}$ depends on $\lambda, \Lambda, \ell$ and $r$. Again by dominated convergence it is obvious that

$$
\frac{1}{\hat{a}^{k}} \underset{k \rightarrow \infty}{\longrightarrow} \frac{1}{\hat{a}}
$$

weakly in $\mathrm{L}^{2}((\ell, r))$. Thus Theorem 3.1 insures that $v_{1}^{k}$ converges to $v_{1}$ weakly in $\mathrm{H}_{0}^{1}((\ell, r))$.

We consider now the continuous versions of the $v_{1}^{k}$ 's and $v_{1}$. By Lemma 4.7,

$$
\forall k \in \mathbb{N},\left\|v_{1}^{k}\right\|_{\infty} \leq C(r-\ell)^{2}
$$

with $C$ depending on $\lambda, \Lambda$, but uniform in $k$. For all $k \in \mathbb{N}$ we have

$$
\forall u, \varphi \in \mathrm{H}_{0}^{1}((\ell, r)),\left\langle-L^{k} u, \varphi\right\rangle_{\mathrm{H}^{-1}\left((\ell, r),\left(\hat{\rho}^{k}\right)^{-1}\right), \mathrm{H}_{0}^{1}\left((\ell, r),\left(\hat{\rho}^{k}\right)^{-1}\right)}=\int_{\ell}^{r} \frac{\hat{a}^{k}}{2} \frac{\mathrm{d} u}{\mathrm{~d} x} \frac{\mathrm{d} \varphi}{\mathrm{d} x} .
$$


The $v_{1}^{k}$ 's are weak solutions of $L^{k} v_{1}^{k}=-v_{0}^{k}$. Using $v_{1}^{k}$ as a test function, (15), (16) and the Poincaré inequality, we get that

$$
\forall k \in \mathbb{N},\left\|v_{1}^{k}\right\|_{\mathrm{H}^{1}((\ell, r))} \leq C^{\prime},
$$

with $C^{\prime}$ depending on $\lambda, \Lambda$, and $r-\ell$ but which is uniform in $k$. By a compact injection argument there exists a subsequence $\left(v_{1}^{k^{\prime}}\right)$ that converges uniformly on $[\ell, r]$ (see Th. VIII.7 in [4]). But its limit is necessarily $v_{1}$, because of the weak convergence in $\mathrm{H}_{0}^{1}((\ell, r))$ of $v_{1}^{k}$ to $v_{1}$. Thus $v_{1}^{k}$ converges uniformly on $[\ell, r]$ to $v_{1}$, and then pointwise.

Step 3. We show that $\mathbb{E}^{x}\left[\tau^{k} \mathbb{1}_{\left\{X_{\tau k}^{k}=r\right\}}\right] \rightarrow \mathbb{E}^{x}\left[\tau \mathbb{1}_{\left\{X_{\tau}=r\right\}}\right]$ as $k \rightarrow \infty$, for all $x \in[\ell, r]$.

Considering (14) it is clear that (7) holds. So combining Theorem 3.3 and Proposition 3.4 we have that $\tau^{k} \mathbb{1}_{\left\{X_{\tau^{k}}^{k}=r\right\}}$ converges in law to $\tau \mathbb{1}_{\left\{X_{\tau}=r\right\}}$.

But by Lemma 4.5 and (13) in Lemma 4.7,

$$
\forall k \in \mathbb{N}, \mathbb{E}^{x}\left[\left(\tau^{k} \mathbb{1}_{\left\{X_{\tau^{k}}^{k}=r\right\}}\right)^{2}\right] \leq K(r-\ell)^{4}
$$

with $K$ depending on $\lambda$ and $\Lambda$ but not on $k$. This ensures that the $\tau^{k} \mathbb{1}_{\left\{X_{\tau^{k}}^{k}=r\right\}}$ 's are uniformly integrable (see in [2] the discussion between Ths. 5.3 and 5.4).

So step 3 is proved.

Taking in account steps 1, 2 and 3 we complete the proof.

We assert now some estimates on $v_{1}$ that will be useful to prove our Donsker theorem.

Proposition 4.8. Set $(L, D(L))=\mathfrak{L}(a, \rho, b)$ with $a, \rho \in \mathfrak{E} \mathfrak{r}(\lambda, \Lambda)$ and $b \in \mathfrak{B}(\Lambda)$. The solution $v_{1}$ of $\mathcal{P}_{1}([\ell, r],+)$ satisfies:

i) For some positive constant $C$ depending only on $\lambda$ and $\Lambda$,

$$
\left\|v_{1}\right\|_{\infty} \leq C(r-\ell)^{2}
$$

ii) For all $x \in(\ell, r)$ and with $\delta:=\min (r-x, x-\ell)$,

$$
v_{1}(x) \geq C^{\prime} \frac{\delta^{4}}{(r-\ell)^{2}}
$$

where $C^{\prime}$ is a positive constant depending only on $\lambda$ and $\Lambda$.

Similar estimates hold for the solution of $\mathcal{P}_{1}([\ell, r],-)$.

Proof. In the proof of Proposition 4.1 we have seen in step 2 that for all $x \in[\ell, r], v_{1}(x)=\lim _{k \rightarrow \infty} v_{1}^{k}(x)$, with the $v_{1}^{k}$ 's defined in step 1.

i) In this proof we have also seen that the $v_{1}^{k}$ 's satisfy (16). Passing to the limit we get $\left|v_{1}(x)\right| \leq C(r-\ell)^{2}$ for all $x \in[\ell, r]$ and $(17)$ is proved.

ii) Choose $x \in(\ell, r)$ fixed. As in the proof of Lemma 4.6 we have for each $v_{1}^{k}$ the representation

$$
\int_{\ell}^{r} G_{[\ell, r]}^{k}(x, y) v_{0}^{k}(y) \exp \left(\Psi^{k}(y)\right)\left(\rho^{k}\right)^{-1}(y) \mathrm{d} y
$$


with the adequate $G_{[\ell, r]}^{k}$ and $\Psi^{k}$. Thanks to (11), $\exp \left( \pm \Psi^{k}\right) \geq 1 / \kappa$ for all $k \in \mathbb{N}$, and, for all $k \in \mathbb{N}$ and all $y \in[\ell, r]$,

$$
\begin{gathered}
\exp \left(\Psi^{k}(y)\right)\left(\rho^{k}\right)^{-1}(y) \geq \frac{1}{\Lambda \kappa}, v_{0}^{k}(y) \geq \frac{\lambda}{\Lambda \kappa^{2}} \cdot \frac{y-\ell}{r-\ell}, \\
G_{[\ell, r]}^{k}(x, y) \geq 2 \frac{\lambda}{\Lambda^{2} \kappa^{3}} \frac{\delta}{r-\ell}(y-\ell) \text { if } y \leq x \\
\text { and } G_{[\ell, r]}^{k}(x, y) \geq 2 \frac{\lambda}{\Lambda^{2} \kappa^{3}} \frac{\delta}{r-\ell}(r-y) \text { if } y \geq x
\end{gathered}
$$

We get finally that $v_{1}^{k}(x) \geq\left(2 \lambda^{2} / 3 \Lambda^{4} \kappa^{6}\right)\left(\delta^{4} /(r-\ell)^{2}\right)$ for all $k$. Passing to the limit we prove (18).

Remark 4.9. Note that the regularization arguments in the proofs of Propositions 4.1 and 4.8 allows us to assert that, in the general case of measurable functions $a, \rho$ and $b$, the Green function associated to the measure $\exp (\Psi(y)) \rho^{-1}(y) \mathrm{d} y$ for elliptic problems on $[\ell, r]$ involving $(L, D(L))$ is $G_{[\ell, r]}$ (with $\Psi$ and $G_{[\ell, r]}$ like in the proof of Lemma 4.6).

Indeed for each $f$ let $u$ be the solution of $(\mathcal{P})$, and a sequence $\left(u^{k}\right)$ constructed to approach $u$ as in step 1 in the proof of Proposition 4.1. For each $k$ we have $u^{k}(x)=\int_{\ell}^{r} G_{[\ell, r]}^{k}(x, y) f(y) \exp \left(\Psi^{k}(y)\right)\left(\rho^{k}\right)^{-1}(y) \mathrm{d} y$. For each $x \in[\ell, r]$ using dominated convergence and passing to the limit we can identify the function $u(x)$ with $\int_{\ell}^{r} G_{[\ell, r]}(x, y) f(y) \exp (\Psi(y)) \rho^{-1}(y) \mathrm{d} y$.

This could have been used to prove Proposition 4.8.

We finally assert an estimate concerning the second order moment.

Proposition 4.10. Assume that $a, \rho \in \mathfrak{E} \mathfrak{r}(\lambda, \Lambda)$ and $b \in \mathfrak{B}(\Lambda)$. Define by $(L, D(L))$ the DFO $\mathfrak{L}(a, \rho, b)$. Let $X$ be the Markov process generated by $(L, D(L))$. Choose $[\ell, r] \subset G$. Set $\tau=\inf \left\{t \geq 0 \mid X_{t} \in\{\ell, r\}\right\}$. For all $x \in[\ell, r]$,

$$
\mathbb{E}^{x}\left[\tau^{2} \mathbb{1}_{\left\{X_{\tau}=r\right\}}\right] \leq K(r-\ell)^{4}
$$

where $K$ is a positive constant depending on $\lambda$ and $\Lambda$.

An estimate similar to (20) holds for $\mathbb{E}^{x}\left[\tau^{2} \mathbb{1}_{\left\{X_{\tau}=\ell\right\}}\right]$.

Proof. As in the proof of Proposition 4.1, we can build a sequence $\left(X^{k}\right)$ of processes generated by smooth coefficients, such that $\left(\tau^{k}\right)^{2} \mathbb{1}_{\left\{X_{\tau k}^{k}=r\right\}}$ converges in law to $\tau^{2} \mathbb{1}_{\left\{X_{\tau}=r\right\}}$. But, as in step 3 in the proof of Proposition 4.1, Lemmas 4.5 and 4.7 , ensure that for all $x \in[\ell, r]$,

$$
\forall k \in \mathbb{N}, \mathbb{E}^{x}\left[\left(\tau^{k}\right)^{2} \mathbb{1}_{\left\{X_{\tau^{k}}^{k}=r\right\}}\right] \leq K(r-\ell)^{4},
$$

with $K$ depending on $\lambda$ and $\Lambda$. So Theorem 5.3 in [2] leads to,

$$
\forall x \in[\ell, r], \mathbb{E}^{x}\left[\tau^{2} \mathbb{1}_{\left\{X_{\tau}=r\right\}}\right] \leq \underset{k}{\liminf } \mathbb{E}^{x}\left[\left(\tau^{k}\right)^{2} \mathbb{1}_{\left\{X_{\tau^{k}}^{k}=r\right\}}\right] \leq K(r-\ell)^{4} .
$$

This concludes the proof.

\section{The ALGORITHM}

Fix $a, \rho \in \mathfrak{E} \mathfrak{l r}(\lambda, \Lambda)$ and $b \in \mathfrak{B}(\Lambda)$. Let $X$ be the process generated by $(L, D(L))=\mathfrak{L}(a, \rho, b)$.

We know present the algorithm, that takes as input arguments (except $a, \rho$ and $b$ ) the starting point $x$ and the time $t$ at which we want an approximation of $X$.

\section{Step 1. Construction of the grid and initialization.}

We first give us a grid $\mathfrak{g}=\left\{x_{j}\right\}_{j \in J}$ on $G$, with $x_{j} \leq x_{j+1}$ for all $j \in J$. 
We assume that $\mathfrak{g}$ has a minimum cell size $h$ and a maximum cell size $H$. As $h$ and $H$ will tend to zero we assume that $H \leq 1$. This corresponds to Condition (8) in Section 4.

For such a grid $\mathfrak{g}$ we define $R:=H / h$ and we call the couple $(h, R)$ the characteristic of $\mathfrak{g}$.

We then initialize the algorithm by setting $\hat{t} \leftarrow 0$ and $S \leftarrow x_{j}$ where $x_{j}$ is the point of $\mathfrak{g}$ that is the closest to $x$.

Step 2. Computation of the transition probabilities and the conditional expectations of exit times.

Let $\left(\tau_{p}^{\mathfrak{g}}\right)_{p \in \mathbb{N}}$ be the sequence of random times defined by

$$
\left\{\begin{array}{l}
\tau_{0}^{\mathfrak{g}}=0 \\
\tau_{p}^{\mathfrak{g}}=\inf \left\{t \geq \tau_{p-1}^{\mathfrak{g}} \mid X_{t} \in \mathfrak{g} \backslash\left\{X_{\tau_{p-1}^{\mathfrak{g}}}\right\}\right\} .
\end{array}\right.
$$

The second step consists in computing for each $x_{j} \in \mathfrak{g}$ :

i) the transition probabilities

$$
\pi(j, j+1):=\mathbb{P}\left[X_{\tau_{p}^{\mathfrak{g}}}=x_{j+1} \mid X_{\tau_{p-1}^{\mathfrak{g}}}=x_{j}\right] .
$$

ii) the conditional expectations

$$
\begin{aligned}
T(j,+) & :=\mathbb{E}\left[\tau_{p}^{\mathfrak{g}}-\tau_{p-1}^{\mathfrak{g}} \mid X_{p-1}=x_{j} ; X_{p}=x_{j+1}\right] \\
& =\mathbb{E}\left[\left(\tau_{p}^{\mathfrak{g}}-\tau_{p-1}^{\mathfrak{g}}\right) \mathbb{1}_{\left\{X_{\tau_{p}^{\mathfrak{g}}}=x_{j+1}\right\}} \mid X_{\tau_{p-1}^{\mathfrak{g}}}=x_{j}\right] / \pi(j, j+1) \\
\text { and } T(j,-) & :=\mathbb{E}\left[\tau_{p}^{\mathfrak{g}}-\tau_{p-1}^{\mathfrak{g}} \mid X_{p-1}=x_{j} ; X_{p}=x_{j-1}\right] \\
& =\mathbb{E}\left[\left(\tau_{p}^{\mathfrak{g}}-\tau_{p-1}^{\mathfrak{g}}\right) \mathbb{1}_{\left\{X_{\tau_{p} \mathfrak{g}}=x_{j-1}\right\}} \mid X_{\tau_{p-1}^{\mathfrak{g}}}=x_{j}\right] /(1-\pi(j, j+1)) .
\end{aligned}
$$

This can be done thanks to the following proposition.

Proposition 5.1. Choose $x_{j} \in \mathfrak{g}$. We have for all $p \in \mathbb{N}^{*}$ :

i) The probability the particle moves from $x_{j}$ to $x_{j+1}$ is

$$
\mathbb{P}\left[X_{\tau_{p}^{\mathfrak{g}}}=x_{j+1} \mid X_{\tau_{p-1}^{\mathfrak{g}}}=x_{j}\right]=\frac{\int_{x_{j-1}}^{x_{j}} \mathrm{e}^{-\Psi(y)} / a(y) \mathrm{d} y}{\int_{x_{j-1}}^{x_{j+1}} \mathrm{e}^{-\Psi(y)} / a(y) \mathrm{d} y},
$$

where

$$
\Psi(x)=2 \int_{x_{j-1}}^{x} \frac{b(y)}{a(y) \rho(y)} \mathrm{d} y, \forall x \in\left[x_{j-1}, x_{j+1}\right] .
$$

ii) The average time to reach a given point is

$$
\mathbb{E}\left[\left(\tau_{p}^{\mathfrak{g}}-\tau_{p-1}^{\mathfrak{g}}\right) \mathbb{1}_{\left\{X_{\tau_{p}^{\mathfrak{g}}}=x_{j+1}\right\}} \mid X_{\tau_{p-1}^{\mathfrak{g}}}=x_{j}\right]=v_{1}\left(x_{j}\right),
$$

where $v_{1}$ is the solution of $\mathcal{P}_{1}\left(\left[x_{j-1}, x_{j+1}\right],+\right)$ as defined in Proposition 4.1, and

$$
\mathbb{E}\left[\left(\tau_{p}^{\mathfrak{g}}-\tau_{p-1}^{\mathfrak{g}}\right) \mathbb{1}_{\left\{X_{\tau_{p} \mathfrak{g}}=x_{j-1}\right\}} \mid X_{\tau_{p-1}^{\mathfrak{g}}}=x_{j}\right]=v_{1}\left(x_{j}\right),
$$

where $v_{1}$ is the solution of $\mathcal{P}_{1}\left(\left[x_{j-1}, x_{j+1}\right],-\right)$ as defined in Proposition 4.1. 
Proof. By the strong Markov property we have for all $p \in \mathbb{N}^{*}$,

$$
\mathbb{P}\left[X_{\tau_{p}^{\mathfrak{g}}}=x_{j+1} \mid X_{\tau_{p-1}^{\mathfrak{g}}}=x_{j}\right]=\mathbb{P}^{x_{j}}\left[X_{\tau_{1}^{\mathfrak{g}}}=x_{j+1}\right]
$$

and

$$
\mathbb{E}\left[\left(\tau_{p}^{\mathfrak{g}}-\tau_{p-1}^{\mathfrak{g}}\right) \mathbb{1}_{\left\{X_{\tau_{p}}^{\mathfrak{g}}=x_{j+1}\right\}} \mid X_{\tau_{p-1}^{\mathfrak{g}}}=x_{j}\right]=\mathbb{E}^{x_{j}}\left[\tau_{1}^{\mathfrak{g}} \mathbb{1}_{\left\{X_{\tau_{1}} \mathfrak{g}=x_{j+1}\right\}}\right] .
$$

Point $\mathrm{i}$ ) is a consequence of Proposition 2.2 and 2.6 and Definition 2.3. We apply Proposition 4.1 to get ii).

Remark 5.2. Note that $\mathbb{P}\left[X_{\tau_{p}^{\mathfrak{g}}}=x_{j+1} \mid X_{\tau_{p-1}^{\mathfrak{g}}}=x_{j}\right]=v_{0}\left(x_{j}\right)$ where $v_{0}$ solves $\mathcal{P}_{0}\left(\left[x_{j-1}, x_{j+1}\right],+\right)$.

It is clear that if we define $\left(S_{p}^{\mathfrak{g}}\right)_{p \in \mathbb{N}}=\left(X_{\tau_{p}^{\mathfrak{g}}}\right)_{p \in \mathbb{N}}$ we get a random walk on the grid $\mathfrak{g}$ with transition probabilities $\mathbb{P}\left[S_{p}^{\mathfrak{g}}=x_{j+1} \mid S_{p-1}^{\mathfrak{g}}=x_{j}\right]=\pi(j, j+1)$.

\section{Step 3. Simulation of the approximating process.}

We compute an approximated value of $X_{t}$, denoted by $\widehat{X}_{t}^{\mathfrak{g}}$, using the following loop.

Initialization: We start with $S=x_{j}$ for some $x_{j} \in \mathfrak{g}$.

(1) Simulate $\operatorname{Ber}(\pi(j, j+1))$.

(2) If success occurs then set, $j \leftarrow j+1, S \leftarrow x_{j+1}$ and $\hat{t} \leftarrow \hat{t}+T(j,+)$.

(3) else set $j \leftarrow j-1, S \leftarrow x_{j-1}$ and $\hat{t} \leftarrow \hat{t}+T(j,-)$.

(4) If $\hat{t}<t$ then go on in the main loop at step (1).

(5) else stop and return $\widehat{X}_{t}^{\mathfrak{g}}=S$.

In the next section we prove that this algorithm converges and evaluate at which speed.

\section{SpeEd OF CONVERGENCE OF THE ALGORITHM}

In this section we will prove the following theorem, that gives us the rate of strong convergence of our scheme.

Theorem 6.1. Assume $a, \rho \in \mathfrak{E} \mathfrak{l l}(\lambda, \Lambda)$ and $b \in \mathfrak{B}(\Lambda)$. Let $X$ be the process generated by $(L, D(L))=$ $\mathfrak{L}(a, \rho, b)$. Fix $0<T<\infty$.

For any grid $\mathfrak{g}$ on $G$, let $\left(\tau_{p}^{\mathfrak{g}}\right)$ be the sequence of stopping times described in Section 5 at step 2. Let us also set

$$
K^{\mathfrak{g}}(t)=\inf \left\{k \in \mathbb{N} \mid \sum_{p=1}^{k} \mathbb{E}\left[\tau_{p}^{\mathfrak{g}}-\tau_{p-1}^{\mathfrak{g}} \mid X_{\tau_{p-1}^{\mathfrak{g}}}, X_{\tau_{p}^{\mathfrak{g}}}\right] \geq t\right\} .
$$

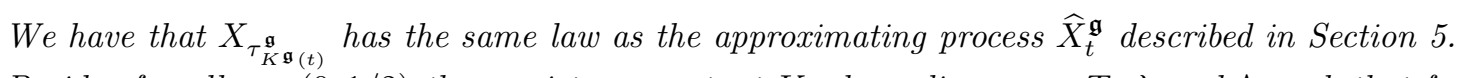

Besides for all $\gamma \in(0,1 / 2)$ there exists a constant $K_{0}$ depending on $\gamma, T, \lambda$ and $\Lambda$ such that for any grid $\mathfrak{g}$ on $G$ of characteristic $(h, R)$,

$$
\forall t \in[0, T], \forall x \in G \mathbb{E}^{x}\left|X_{\tau_{K \mathfrak{g}(t)}^{\mathfrak{g}}}-X_{t}\right| \leq K_{0} C_{0}(R) h^{\gamma}
$$

where $C_{0}(\cdot)$ is a positive and increasing function of $R$.

To prove Theorem 6.1 we need a few lemmas. For all grid $\mathfrak{g}$ we call $S^{\mathfrak{g}}$ the random walk on $\mathfrak{g}$ defined by $S_{p}^{\mathfrak{g}}=X_{\tau_{p}^{\mathfrak{g}}}$ for all $p \in \mathbb{N}$.

Lemma 6.2. For all $\gamma \in(0,1 / 2)$ and all $1<p<4 / 3$ there exists a constant $C_{p}^{\gamma}$ depending on $\gamma, p, \lambda, \Lambda$ and $T$, such that

$$
\mathbb{E}\left(\sup _{s \neq t, s, t \in[0, T]} \frac{\left|X_{t}-X_{s}\right|}{|t-s|^{\gamma}}\right)^{p} \leq C_{p}^{\gamma}<\infty .
$$


To prove this lemma we will use Lemma 2.8 and the technique used by one of the authors in [14] to prove his Lemma 3.

Proof.

Step 1. We prove that for any $\alpha>2$ there is a constant $B$ depending on $\lambda, \Lambda$ and $T$ such that for all $0 \leq s \leq t \leq T$

With the Markov property,

$$
\sup _{x \in G} \mathbb{E}^{x}\left[\sup _{r \in[s, t]}\left|X_{r}-X_{s}\right|^{\alpha}\right] \leq B|t-s|^{\alpha / 2}
$$

$$
\sup _{x \in G} \mathbb{E}^{x}\left[\sup _{r \in[s, t]}\left|X_{r}-X_{s}\right|^{\alpha}\right]=\sup _{x \in G} \mathbb{E}^{x}\left[\sup _{r \in[0, t-s]}\left|X_{r}-x\right|^{\alpha}\right] .
$$

Fix $\alpha>2$ and $t \in[0, T]$. For $x \in G$ and $d>0$ let us define the exit time $\tau=\inf \left\{r \geq 0 \mid X_{r} \notin(x-d, x+d)\right\}$. Using $(a+b)^{\alpha} \leq 2^{\alpha-1}\left(a^{\alpha}+b^{\alpha}\right)$ and the strong Markov property we get,

$$
\begin{aligned}
& \sup _{x \in G} \mathbb{E}^{x}\left[\sup _{r \in[0, t]}\left|X_{r}-x\right|^{\alpha}\right] \leq d^{\alpha}+\sup _{x \in G} \mathbb{E}^{x}\left[\sup _{r \in[0, t]}\left|X_{r}-x\right|^{\alpha} \mathbb{1}_{\{t>\tau\}}\right] \leq d^{\alpha}+\sup _{x \in G} \mathbb{E}^{x}\left[\sup _{r \in[\tau, t]}\left|X_{r}-x\right|^{\alpha} \mathbb{1}_{\{t>\tau\}}\right] \\
& \leq d^{\alpha}+2^{\alpha-1}\left(d^{\alpha}+\sup _{x \in G} \mathbb{E}^{x}\left[\sup _{r \in[\tau, t]}\left|X_{r}-X_{\tau}\right|^{\alpha} \mathbb{1}_{\{t>\tau\}}\right]\right. \\
& \leq\left(2^{\alpha-1}+1\right) d^{\alpha}+2^{\alpha-1} \sup _{x \in G} \mathbb{E}^{x}\left[\sup _{r \in[0, t]}\left|X_{r}-x\right|^{\alpha}\right] \sup _{x \in G} \mathbb{P}^{x}[t>\tau] .
\end{aligned}
$$

Thanks to Lemma 2.8 we have $\sup _{x \in G} \mathbb{P}^{x}[t>\tau] \leq A \exp \left(-A d^{2} / t\right)$. Hence step 1 is proved if we take $d$ equal to $\mu \sqrt{t}$ with $\mu$ large enough so that we have $2^{\alpha-1} A \exp \left(-A \mu^{2}\right) \leq 1 / 2$, and if we finally replace $t$ by $t-s$.

Step 2. Choose $\gamma \in(0,1 / 2)$. There exists $\alpha>2$ such that $0<\gamma<1 / 2-1 / \alpha$. Combining step 1 and the Kolmogorov-Čentsov theorem (see [20]),

$$
\mathbb{E}\left(\sup _{s \neq t, s, t \in[0, T]} \frac{\left|X_{t}-X_{s}\right|}{|t-s|^{\gamma}}\right)^{\alpha} \leq C_{\alpha}^{\gamma}<\infty
$$

with $C_{\alpha}^{\gamma}$ depending on $\gamma, \alpha, \lambda, \Lambda$ and $T$.

As $\alpha>2$ it then suffices to use the Jensen inequality to complete the proof.

Lemma 6.3. In the context of Theorem 6.1, the $\left(\tau_{p}^{\mathfrak{g}}-\tau_{p-1}^{\mathfrak{g}}\right)$ 's are independent conditionally to the paths of $S^{\mathfrak{g}}$. Proof. Tedious but straightforward.

Lemma 6.4. There are constants $M, M^{\prime}$ and $M^{\prime \prime}$ depending on $\lambda$ and $\Lambda$ such that, for any grid $\mathfrak{g}$ on $G$ of characteristic $(h, R)$, in the context of Theorem 6.1,

$$
\begin{aligned}
& \text { i) } \forall p \in \mathbb{N}, \forall x_{j} \in \mathfrak{g}, \quad \frac{M^{\prime}}{R^{2}} h^{2} \leq \mathbb{E}\left[\tau_{p}^{\mathfrak{g}}-\tau_{p-1}^{\mathfrak{g}} \mid X_{\tau_{p-1}^{\mathfrak{g}}}^{\mathfrak{g}}=x_{j} ; X_{\tau_{p}^{\mathfrak{g}}}^{\mathfrak{g}}=x_{j \pm 1}\right] \leq M R^{3} h^{2} . \\
& \text { ii) } \forall p \in \mathbb{N}, \forall x_{j} \in \mathfrak{g}, \quad \operatorname{Var}\left[\tau_{p}^{\mathfrak{g}}-\tau_{p-1}^{\mathfrak{g}} \mid X_{\tau_{p-1}^{\mathfrak{g}}}^{\mathfrak{g}}=x_{j} ; X_{\tau_{p}^{\mathfrak{g}}}^{\mathfrak{g}}=x_{j \pm 1}\right] \leq M^{\prime \prime} R^{4} h^{4} .
\end{aligned}
$$

Proof. Remember that for all $x_{j} \in \mathfrak{g}, h \leq x_{j+1}-x_{j} \leq R h$. To prove Point i) it then suffices to use Propositions 4.8 and 5.1, Remark 5.2 and the second line of (19). Point ii) is a direct consequence of (20) in Proposition 4.10 .

Lemma 6.5. In the context of Theorem 6.1 there exists a constant $K$ depending on $T, \lambda$ and $\Lambda$ such that for any grid $\mathfrak{g}$ of characteristic $(h, R)$,

$$
\mathbb{E}\left|\tau_{K \mathfrak{g}(t)}^{\mathfrak{g}}-t\right|^{2} \leq K C_{1}(R) h^{2}
$$

where $C_{1}(\cdot)$ is a positive and increasing function of $R$. 
Proof. Let $\mathfrak{g}$ be a grid of characteristic $(h, R)$. Using $(a+b)^{2} \leq 2\left(a^{2}+b^{2}\right)$ we have,

$\mathbb{E}\left|\tau_{K}^{\mathfrak{g}}(t)-t\right|^{2} \leq 2 \mathbb{E}\left|\sum_{p=1}^{K^{\mathfrak{g}}(t)} \mathbb{E}\left[\tau_{p}^{\mathfrak{g}}-\tau_{p-1}^{\mathfrak{g}} \mid X_{\tau_{p-1}^{\mathfrak{g}}}, X_{\tau_{p}^{\mathfrak{g}}}\right]-t\right|^{2}+2 \mathbb{E}\left|\sum_{p=1}^{K^{\mathfrak{g}}(t)} \mathbb{E}\left[\tau_{p}^{\mathfrak{g}}-\tau_{p-1}^{\mathfrak{g}} \mid X_{\tau_{p-1}^{\mathfrak{g}}}, X_{\tau_{p}^{\mathfrak{g}}}\right]-\tau_{K^{\mathfrak{g}}(t)}^{\mathfrak{g}}\right|^{2}$.

By definition of $K^{\mathfrak{g}}(t)$ and the i) of Lemma 6.4 it is obvious that

$$
\left|\sum_{p=1}^{K^{\mathfrak{g}}(t)} \mathbb{E}\left[\tau_{p}^{\mathfrak{g}}-\tau_{p-1}^{\mathfrak{g}} \mid X_{\tau_{p-1}^{\mathfrak{g}}}, X_{\tau_{p}^{\mathfrak{g}}}\right]-t\right| \leq \max _{x_{j} \in \mathfrak{g}} \mathbb{E}\left[\tau_{p}^{\mathfrak{g}}-\tau_{p-1}^{\mathfrak{g}} \mid X_{\tau_{p-1}^{\mathfrak{g}}}=x_{j}, X_{\tau_{p}^{\mathfrak{g}}}=x_{j \pm 1}\right] \leq M R^{3} h^{2} \text { a.s. }
$$

and we thus get an estimate of the first term on the right in (21):

$$
\mathbb{E}\left|\sum_{p=1}^{K^{\mathfrak{g}}(t)} \mathbb{E}\left[\tau_{p}^{\mathfrak{g}}-\tau_{p-1}^{\mathfrak{g}} \mid X_{\tau_{p-1}^{\mathfrak{g}}}, X_{\tau_{p}^{\mathfrak{g}}}\right]-t\right|^{2} \leq M^{2} R^{6} h^{4}
$$

We will now estimate the second term. We have,

$$
\mathbb{E}\left|\sum_{p=1}^{K^{\mathfrak{g}}(t)} \mathbb{E}\left[\tau_{p}^{\mathfrak{g}}-\tau_{p-1}^{\mathfrak{g}} \mid X_{\tau_{p-1}^{\mathfrak{g}}}, X_{\tau_{p}^{\mathfrak{g}}}\right]-\tau_{K \mathfrak{g}(t)}^{\mathfrak{g}}\right|^{2}=\mathbb{E} \mid \sum_{p=1}^{K^{\mathfrak{g}}(t)}\left(\tau_{p}^{\mathfrak{g}}-\tau_{p-1}^{\mathfrak{g}}-\left.\mathbb{E}\left[\tau_{p}^{\mathfrak{g}}-\tau_{p-1}^{\mathfrak{g}} \mid X_{\tau_{p-1}^{\mathfrak{g}}}, X_{\tau_{p}^{\mathfrak{g}}}\right]\right|^{2}\right.
$$

For some $k \in \mathbb{N}$ there are paths of $S^{\mathfrak{g}}$ such that $K^{\mathfrak{g}}(t)=k$ and $\mathbb{P}[$ path $] \neq 0$. So we can write

$$
\begin{aligned}
\mathbb{E} \mid \sum_{p=1}^{K^{\mathfrak{g}}(t)}\left(\tau_{p}^{\mathfrak{g}}-\tau_{p-1}^{\mathfrak{g}}-\mathbb{E}\left[\tau_{p}^{\mathfrak{g}}-\tau_{p-1}^{\mathfrak{g}} \mid\right.\right. & \left.\left.X_{\tau_{p-1}^{\mathfrak{g}}}, X_{\tau_{p}^{\mathfrak{g}}}\right]\right)\left.\right|^{2} \\
& =\sum_{k \in \mathbb{N}} \mathbb{E}\left[\mathbb{1}_{\left\{K^{\mathfrak{g}}(t)=k\right\}}\left|\sum_{p=1}^{k}\left(\tau_{p}^{\mathfrak{g}}-\tau_{p-1}^{\mathfrak{g}}-\mathbb{E}\left[\tau_{p}^{\mathfrak{g}}-\tau_{p-1}^{\mathfrak{g}} \mid X_{\tau_{p-1}^{\mathfrak{g}}}, X_{\tau_{p}^{\mathfrak{g}}}\right]\right)\right|^{2}\right]
\end{aligned}
$$

But for each $k$,

$$
\begin{aligned}
\mathbb{E}\left[\mathbb{1}_{\left\{K^{\mathfrak{g}}(t)=k\right\}}\left|\sum_{p=1}^{k}\left(\tau_{p}^{\mathfrak{g}}-\tau_{p-1}^{\mathfrak{g}}-\mathbb{E}\left[\tau_{p}^{\mathfrak{g}}-\tau_{p-1}^{\mathfrak{g}} \mid X_{\tau_{p-1}^{\mathfrak{g}}}, X_{\tau_{p}^{\mathfrak{g}}}\right]\right)\right|^{2}\right] \\
=\sum_{\text {paths s.t. } K^{\mathfrak{g}}(t)=k} \mathbb{E}\left[\mathbb{1}_{\{\text {path }\}}\left|\sum_{p=1}^{k}\left(\tau_{p}^{\mathfrak{g}}-\tau_{p-1}^{\mathfrak{g}}-\mathbb{E}\left[\tau_{p}^{\mathfrak{g}}-\tau_{p-1}^{\mathfrak{g}} \mid X_{\tau_{p-1}^{\mathfrak{g}}}, X_{\tau_{p}^{\mathfrak{g}}}\right]\right)\right|^{2}\right] \\
\left.=\sum_{\text {paths s.t. } K^{\mathfrak{g}}(t)=k} \mathbb{E}\left[\mathbb{1}_{\{\text {path }\}} \operatorname{Var}\left[\sum_{p=1}^{k}\left(\tau_{p}^{\mathfrak{g}}-\tau_{p-1}^{\mathfrak{g}}\right) \mid \text { path }\right] \text { (path }\right)\right] .
\end{aligned}
$$

We use now Lemma 6.3, which allows us to assert that

$$
\operatorname{Var}\left[\sum_{p=1}^{k}\left(\tau_{p}^{\mathfrak{g}}-\tau_{p-1}^{\mathfrak{g}}\right) \mid \text { path }\right]=\sum_{p=1}^{k} \operatorname{Var}\left[\tau_{p}^{\mathfrak{g}}-\tau_{p-1}^{\mathfrak{g}} \mid \text { path }\right],
$$


and the ii) of Lemma 6.4. Thus,

$$
\begin{array}{r}
\mathbb{E}\left[\mathbb{1}_{\left\{K^{\mathfrak{g}}(t)=k\right\}}\left|\sum_{p=1}^{k}\left(\tau_{p}^{\mathfrak{g}}-\tau_{p-1}^{\mathfrak{g}}-\mathbb{E}\left[\tau_{p}^{\mathfrak{g}}-\tau_{p-1}^{\mathfrak{g}} \mid X_{\tau_{p-1}^{\mathfrak{g}}}, X_{\tau_{p}^{\mathfrak{g}}}\right]\right)\right|^{2}\right] \leq \sum_{\text {paths s.t. } K^{\mathfrak{g}}(t)=k} k M^{\prime \prime} R^{4} h^{4} \mathbb{E}\left[\mathbb{1}_{\{\text {path }\}}\right] \\
=M^{\prime \prime} R^{4} h^{4} k \mathbb{P}\left[K^{\mathfrak{g}}(t)=k\right]
\end{array}
$$

Taking in account (23), (24) and (25) we then have,

$$
\mathbb{E}\left|\sum_{p=1}^{K^{\mathfrak{g}}(t)} \mathbb{E}\left[\tau_{p}^{\mathfrak{g}}-\tau_{p-1}^{\mathfrak{g}} \mid X_{\tau_{p-1}^{\mathfrak{g}}}, X_{\tau_{p}^{\mathfrak{g}}}\right]-\tau_{K \mathfrak{g}^{\mathfrak{g}}(t)}^{\mathfrak{g}}\right|^{2} \leq M^{\prime \prime} R^{4} h^{4} \mathbb{E}\left[K^{\mathfrak{g}}(t)\right] .
$$

It remains to evaluate $\mathbb{E}\left[K^{\mathfrak{g}}(t)\right]$. But, by definition of $K^{\mathfrak{g}}(t)$ and the i) of Lemma 6.4 , for all $t \in[0, T]$

$$
K^{\mathfrak{g}}(t) \leq\left\lfloor\frac{t}{\min _{x_{j} \in \mathfrak{g}} \mathbb{E}\left[\tau_{p}^{\mathfrak{g}}-\tau_{p-1}^{\mathfrak{g}} \mid X_{\tau_{p-1}^{\mathfrak{g}}}=x_{j}, X_{\tau_{p}^{\mathfrak{g}}}=x_{j \pm 1}\right]}\right\rfloor+1 \leq \frac{T}{M^{\prime}} \frac{R^{2}}{h^{2}}+1 \text { a.s. }
$$

Using this and (26) we get

$$
\mathbb{E}\left|\sum_{p=1}^{K^{\mathfrak{g}}(t)} \mathbb{E}\left[\tau_{p}^{\mathfrak{g}}-\tau_{p-1}^{\mathfrak{g}} \mid X_{\tau_{p-1}^{\mathfrak{g}}}, X_{\tau_{p}^{\mathfrak{g}}}\right]-\tau_{K^{\mathfrak{g}}(t)}^{\mathfrak{g}}\right|^{2} \leq \frac{T M^{\prime \prime}}{M^{\prime}} R^{6} h^{2}+M^{\prime \prime} R^{4} h^{4} .
$$

Taking in account (22) and (27) and the fact that $h \leq 1$ (see (8) and step 1 in Section 5) we complete the proof.

Proof of Theorem 6.1. That $X_{\tau_{K}^{\mathfrak{g}}(t)}$ has the same law as $\widehat{X}_{t}^{\mathfrak{g}}$ is clear.

Let us choose $\gamma \in(0,1 / 2)$ and set $q=2 / \gamma$. Let $p$ be the conjugate of $q$. We have $1<p<4 / 3$. By the Hölder inequality we can write for any grid $\mathfrak{g}$ of characteristic $(h, R)$ and any $t \in[0, T]$,

$$
\mathbb{E}\left|X_{\tau_{K}^{\mathfrak{g}}(t)}-X_{t}\right| \leq\left(\mathbb{E}\left[\sup _{s \neq t} \frac{\left|X_{t}-X_{s}\right|}{|t-s|^{\gamma}}\right]^{p}\right)^{1 / p} \cdot\left(\mathbb{E}\left|\tau_{K \mathfrak{g}(t)}^{\mathfrak{g}}-t\right|^{2}\right)^{1 / q}
$$

By Lemma 6.2 and Lemma 6.5 we get

$$
\mathbb{E}\left|X_{\tau_{K \mathfrak{g}(t)}^{\mathfrak{g}}}-X_{t}\right| \leq C(\gamma)\left(K C_{1}(R) h^{2}\right)^{1 / q}=C(\gamma) K^{\gamma / 2}\left(C_{1}(R)\right)^{\gamma / 2} h^{\gamma},
$$

where the constant $C(\gamma) K^{\gamma / 2}$ depends on $\gamma, T, \lambda$ and $\Lambda$ but not on the grid. The proof is now complete.

\section{Simulations}

One can wonder how to practically make the computations in step 2 of the algorithm. In fact we can first construct piecewise approximations $\widetilde{a}, \widetilde{\rho}, \widetilde{b}$ of the given coefficients $a, \rho$ and $b$. We can then approach the process generated by $\mathfrak{L}(\widetilde{a}, \widetilde{\rho}, \widetilde{b})$, by another one for which the computations in step 2 are easy to make. 


\subsection{The choice of the piecewise constant coefficients}

In many practical situations $G$ is bounded and the coefficients $a, \rho$ and $b$ can actually be considered as being right continuous with left limit (r.c.l.l.), and of class $\mathrm{C}^{1}$ except on a set $\mathcal{I}$ of points of finite cardinal.

Then we can choose the grid $\mathfrak{g}$ of characteristic $(h, R)$ such that $\mathcal{I} \subset \mathfrak{g}$ and define r.c.l.l. piecewise constant coefficients $\widetilde{a}, \widetilde{\rho}$ and $\widetilde{b}$, by $\widetilde{a}(x)=a(x), \widetilde{\rho}(x)=\rho(x)$ and $\widetilde{b}(x)=b(x)$ for all $x \in \mathfrak{g}$.

Using the mean value theorem it is then easy to show that the coefficients $\widetilde{a}, \widetilde{\rho}$ and $\widetilde{b}$ tend pointwise respectively to $a, \rho$ and $b$ as $h$ tends to zero. By dominated convergence and Theorem 3.3 , the process generated by $\mathfrak{L}(\widetilde{a}, \widetilde{\rho}, \widetilde{b})$ then converges in law to the one generated by $\mathfrak{L}(a, \rho, b)$ (using a similar technique to the one in the proof of Proposition 7.2 below, we could even show the weak error introduced by this approximation is of order $\mathcal{O}(h)$, that is less than the one of our scheme).

On the other hand, our algorithm also allows us to perform some change of scale, and then to catch directly some small scale behavior of the process, by considering that the grid is constructed at a mesoscopic scale. The homogenization theory can then be applied to deal with the local behavior of the coefficients. The article [18] perform the construction of a Markov chain that approximate a diffusion in dimension 2 or 3 in presence of locally irregular coefficients, but they need to solve a finite volume problem. In our simpler case, we construct the random walk by taking into account only local informations.

To be more precise, in some practical situation, one can have very irregular but locally periodic coefficients, with rapid oscillations. In this situation the theory of $G$-convergence can help us the get homogenized coefficients that will be piecewise constant (see [24], see also [1] on homogenization).

To fix the ideas, define by $\mathcal{I}=\left\{x_{i}\right\}_{i \in I}$ a set of points in $G$ and let us consider $\hat{a} \in \mathfrak{E} \mathfrak{r r}(\lambda, \Lambda)$ that is periodic on each interval $\left[x_{i}, x_{i+1}\right]$. Let the coefficient $a$ be defined by $a(x)=\hat{a}\left(x / \varepsilon_{i}\right), \forall x \in\left[x_{i}, x_{i+1}\right]$, with a very small coefficient $\varepsilon_{i}$ on each interval $\left[x_{i}, x_{i+1}\right]$. The coefficient $a$ is rapidly oscillating. Suppose we want to simulate the paths of the process $X$ generated by $\mathfrak{L}(a, 1,0)$. It can be shown that, on each $\left[x_{i}, x_{i+1}\right]$,

$$
\frac{1}{\hat{a}\left(\cdot / \varepsilon_{i}\right)} \frac{\mathrm{L}^{2}}{\varepsilon_{i} \downarrow 0} \int_{x_{i}}^{x_{i+1}} \frac{\mathrm{d} y}{\hat{a}(y)} .
$$

So we define a coefficient $\widetilde{a}$, constant on each $\left[x_{i}, x_{i+1}\right)$, by,

$$
\widetilde{a}\left(x_{i}\right)=\left(\int_{x_{i}}^{x_{i+1}} \frac{\mathrm{d} y}{\hat{a}(y)}\right)^{-1}
$$

in order that, on each $\left[x_{i}, x_{i+1}\right], 1 / \hat{a}\left(. / \varepsilon_{i}\right)$ converges weakly in $\mathrm{L}^{2}$ to $(1 / \widetilde{a})$.

As the $\varepsilon_{i}$ are very small, by theorem 3.3 we can consider that the process $\widetilde{X}$ generated by $\mathfrak{L}(\widetilde{a}, 1,0)$ is a good weak approximation of $X$. In the same manner we could homogenize the coefficients $\rho$ and $b$, if needed.

\subsection{Approximation of the process generated by piecewise constant coefficients}

Assume that $G$ is bounded. Let $\mathfrak{g}$ be a grid on $G$ of characteristic $(h, R)$. Let us assume that $\widetilde{a}, \widetilde{\rho} \in \mathfrak{E} \mathfrak{r r}(\lambda, \Lambda)$ and $\widetilde{b} \in \mathfrak{B}(\Lambda)$ are right continuous with left limit and are constant on each cell $\left[x_{j}, x_{j+1}\right)$ of $\mathfrak{g}$. Let $\widetilde{X}$ be the process generated by $\mathfrak{L}(\widetilde{a}, \widetilde{\rho}, \widetilde{b})$. Fix $x_{0} \in G$ and

$$
\Psi(x)=\int_{x_{0}}^{x} \frac{\widetilde{b}(y)}{\widetilde{a}(y) \widetilde{\rho}(y)} \mathrm{d} y, \forall x \in G .
$$

Let $\Psi^{\mathfrak{g}}$ be right continuous with left limit, constant on each cell $\left[x_{j}, x_{j+1}\right)$ of $\mathfrak{g}$, and defined by $\Psi^{\mathfrak{g}}\left(x_{j}\right)=\Psi\left(x_{j}\right)$ for all $x_{j} \in \mathfrak{g}$.

Let $X^{\mathfrak{g}}$ be the process generated by $\mathfrak{L}\left(\widetilde{a} \mathrm{e}^{\Psi^{\mathfrak{g}}}, \widetilde{\rho} \mathrm{e}^{-\Psi^{\mathfrak{g}}}, 0\right)$. In this context we have the two following propositions. 
Proposition 7.1. Fix $x_{j} \in \mathfrak{g}$. Set $l=x_{j+1}-x_{j}, \ell^{\prime}=x_{j}-x_{j-1}$ and $c=\exp \left(2 \widetilde{b}\left(x_{j-1}\right) \ell^{\prime} /\left[\widetilde{a}\left(x_{j-1}\right) \widetilde{\rho}\left(x_{j-1}\right)\right]\right)$. For all $p \in \mathbb{N}^{*}$ :

i) The probability the particle moves from $x_{j}$ to $x_{j+1}$ is

$$
\mathbb{P}\left[X_{\tau_{p}^{\mathfrak{g}}}^{\mathfrak{g}}=x_{j+1} \mid X_{\tau_{p-1}^{\mathfrak{g}}}^{\mathfrak{g}}=x_{j}\right]=\frac{\widetilde{a}\left(x_{j}\right) \ell^{\prime}}{\widetilde{a}\left(x_{j}\right) \ell^{\prime}+\widetilde{a}\left(x_{j-1}\right) \ell / c}
$$

ii) The averages of the reaching time of the next position of the particle on the grid are given by

$$
\begin{aligned}
\mathbb{E} & {\left[\left(\tau_{p}^{\mathfrak{g}}-\tau_{p-1}^{\mathfrak{g}}\right) \mathbb{1}_{\left\{X_{\tau_{p}^{\mathfrak{g}}}^{\mathfrak{g}}=x_{j+1}\right\}} \mid X_{\tau_{p-1}^{\mathfrak{g}}}^{\mathfrak{g}}=x_{j}\right] } \\
& =\frac{1}{\left(\widetilde{a}\left(x_{j-1}\right) l+\widetilde{a}\left(x_{j}\right) \ell^{\prime} c\right)^{2}}\left[\frac{1}{3} \frac{\widetilde{a}\left(x_{j-1}\right)}{\widetilde{\rho}\left(x_{j}\right)} c \ell^{3} \ell^{\prime}+\frac{\widetilde{a}\left(x_{j}\right)}{\widetilde{\rho}\left(x_{j}\right)} c^{2} l^{2}\left(\ell^{\prime}\right)^{2}+\frac{2}{3} \frac{\widetilde{a}\left(x_{j}\right)}{\widetilde{\rho}\left(x_{j-1}\right)} c \ell\left(\ell^{\prime}\right)^{3}\right] \\
\text { and } \mathbb{E} & {\left[\left(\tau_{p}^{\mathfrak{g}}-\tau_{p-1}^{\mathfrak{g}}\right) \mathbb{1}_{\left\{X_{\tau_{p}^{\mathfrak{g}}}^{\mathfrak{g}}=x_{j-1}\right\}} \mid X_{\tau_{p-1}^{\mathfrak{g}}}^{\mathfrak{g}}=x_{j}\right] } \\
& =\frac{1}{\left(\widetilde{a}\left(x_{j-1}\right) \ell+\widetilde{a}\left(x_{j}\right) \ell^{\prime} c\right)^{2}}\left[\frac{2}{3} \frac{\widetilde{a}\left(x_{j}\right)}{\widetilde{\rho}\left(x_{j}\right)} c \ell^{3} \ell^{\prime}+\frac{\widetilde{a}\left(x_{j-1}\right)}{\widetilde{\rho}\left(x_{j-1}\right)} \ell^{2}\left(\ell^{\prime}\right)^{2}+\frac{1}{3} \frac{\widetilde{a}\left(x_{j}\right)}{\widetilde{\rho}\left(x_{j-1}\right)} c \ell\left(\ell^{\prime}\right)^{3}\right] .
\end{aligned}
$$

Proof. To prove the point i) it suffices to compute $\left(s\left(x_{j}\right)-s\left(x_{j-1}\right)\right) /\left(s\left(x_{j+1}\right)-s\left(x_{j-1}\right)\right)$ with the scale function $s$ defined by (5) with $a$ replaced by $\widetilde{a} \mathrm{e}^{\Psi^{\mathfrak{g}}}$.

To prove the point ii) let us consider the process $\bar{X}$ generated by $(\bar{L}, D(\bar{L})):=\mathfrak{L}(\bar{a}, \bar{\rho}, 0)$ with

$$
\bar{a}(x)= \begin{cases}\widetilde{a}\left(x_{j-1}\right) \mathrm{e}^{\Psi^{\mathfrak{g}}\left(x_{j-1}\right)} & \text { if } x<0 \\ \widetilde{a}\left(x_{j}\right) \mathrm{e}^{\Psi^{\mathfrak{g}}\left(x_{j}\right)} & \text { if } x \geq 0\end{cases}
$$

and

$$
\bar{\rho}(x)= \begin{cases}\widetilde{\rho}\left(x_{j-1}\right) \mathrm{e}^{-\Psi^{\mathfrak{g}}\left(x_{j-1}\right)} & \text { if } x<0, \\ \widetilde{\rho}\left(x_{j}\right) \mathrm{e}^{-\psi^{\mathfrak{g}}\left(x_{j}\right)} & \text { if } x \geq 0\end{cases}
$$

and set $\tau=\inf \left\{t \geq 0 \mid \bar{X}_{t} \in\left\{-\ell^{\prime}, \ell\right\}\right\}$. By the definitions of $X^{\mathfrak{g}}$ and $\bar{X}$ and the strong Markov property it is clear that,

$$
\mathbb{E}^{0}\left[\tau \mathbb{1}_{\left\{X_{\tau}=\ell\right\}}\right]=\mathbb{E}\left[\left(\tau_{p}^{\mathfrak{g}}-\tau_{p-1}^{\mathfrak{g}}\right) \mathbb{1}_{\left\{X_{\tau_{p}^{\mathfrak{g}}}^{\mathfrak{g}}=x_{j+1}\right\}} \mid X_{\tau_{p-1}^{\mathfrak{g}}}^{\mathfrak{g}}=x_{j}\right]
$$

But proposition 4.1 asserts that $\mathbb{E}^{0}\left[\tau \mathbb{1}_{\left\{X_{\tau}=\ell\right\}}\right]=v_{1}(0)$ for the solution $v_{1} \in D(\bar{L}) \cap \mathrm{C}$ to

$$
\left\{\begin{array}{l}
\frac{1}{2} \widetilde{\rho}\left(x_{j-1}\right) \widetilde{a}\left(x_{j-1}\right) v_{1}^{\prime \prime}(x)=-v_{0}(x) \text { for } x \in\left[-\ell^{\prime}, 0\right), \\
\frac{1}{2} \widetilde{\rho}\left(x_{j}\right) \widetilde{a}\left(x_{j}\right) v_{1}^{\prime \prime}(x)=-v_{0}(x) \text { for } x \in[0, l], \\
v_{1}(0-)=v_{1}(0+), \\
\widetilde{a}\left(x_{j-1}\right) \mathrm{e}^{\Psi^{\mathfrak{g}}\left(x_{j-1}\right)} v_{1}^{\prime}(0-)=\widetilde{a}\left(x_{j}\right) \mathrm{e}^{\Psi^{\mathfrak{g}}\left(x_{j}\right)} v_{1}^{\prime}(0+), \\
v_{1}^{\prime}\left(-\ell^{\prime}\right)=v_{1}^{\prime}(\ell)=0,
\end{array}\right.
$$


where $v_{0}$ is itself in $D(\bar{L}) \cap \mathrm{C}$ and solves

$$
\left\{\begin{array}{l}
\frac{1}{2} \widetilde{\rho}\left(x_{j-1}\right) \widetilde{a}\left(x_{j-1}\right) v_{0}^{\prime \prime}(x)=0 \text { for } x \in\left[-\ell^{\prime}, 0\right), \\
\frac{1}{2} \widetilde{\rho}\left(x_{j}\right) \widetilde{a}\left(x_{j}\right) v_{0}^{\prime \prime}(x)=0 \text { for } x \in[0, l] \\
v_{0}(0-)=v_{0}(0+), \\
\widetilde{a}\left(x_{j-1}\right) \mathrm{e}^{\Psi^{\mathfrak{g}}\left(x_{j-1}\right)} v_{0}^{\prime}(0-)=\widetilde{a}\left(x_{j}\right) \mathrm{e}^{\Psi^{\mathfrak{g}}\left(x_{j}\right)} v_{0}^{\prime}(0+), \\
v_{0}^{\prime}\left(-\ell^{\prime}\right)=0 \\
v_{0}^{\prime}(\ell)=1
\end{array}\right.
$$

Note that in both systems the third line yields the continuity of the solution, while the fourth line indicates that it belongs to $D(\bar{L})$.

So we compute first $v_{0}$ and get a function of the form $A x+B$ on $\left[-\ell^{\prime}, 0\right)$ and $B x+C$ on $[0, l]$. Then we compute $v_{1}$ and eventually $v_{1}(0)$ that gives us $\mathbb{E}\left[\left(\tau_{p}^{\mathfrak{g}}-\tau_{p-1}^{\mathfrak{g}}\right) \mathbb{1}_{\left\{X_{\tau_{p}^{\mathfrak{g}}}^{\mathfrak{g}}=x_{j+1}\right\}} \mid X_{\tau_{p-1}^{\mathfrak{g}}}^{\mathfrak{g}}=x_{j}\right]$.

We proceed in the same manner for computing

$$
\mathbb{E}\left[\left(\tau_{p}^{\mathfrak{g}}-\tau_{p-1}^{\mathfrak{g}}\right) \mathbb{1}_{\left\{X_{\tau_{p}^{\mathfrak{g}}}^{\mathfrak{g}}=x_{j-1}\right\}} \mid X_{\tau_{p-1}^{\mathfrak{g}}}^{\mathfrak{g}}=x_{j}\right]
$$

and we get the second formula of ii).

Proposition 7.2. Let $f$ be in $\mathrm{H}_{0}^{1}(G) \cap \mathrm{C}^{0}(G)$. There exists a constant $C$ depending on $T, \lambda, \Lambda, G,\|f\|_{\infty}$ and $\|\mathrm{d} f / \mathrm{d} x\|_{2}$, such that

$$
\sup _{(t, x) \in[0, T] \times G}\left|\mathbb{E}^{x} f\left(\widetilde{X}_{t}\right)-\mathbb{E}^{x} f\left(X_{t}^{\mathfrak{g}}\right)\right| \leq C R h^{1 / 2} .
$$

Proof. We can consider that $\tilde{X}$ is generated by $\mathfrak{L}\left(\widetilde{a} \mathrm{e}^{\Psi}, \widetilde{\rho} \mathrm{e}^{-\Psi}, 0\right)$. The functions $\mathbb{E}^{x} f\left(\widetilde{X}_{t}\right)$ and $\mathbb{E}^{x} f\left(X_{t}^{\mathfrak{g}}\right)$ are continuous versions of the solutions to parabolic PDEs with initial condition $f$ involving respectively the operators $\mathfrak{L}\left(\widetilde{a} \mathrm{e}^{\Psi}, \widetilde{\rho} \mathrm{e}^{-\Psi}, 0\right)$ and $\mathfrak{L}\left(\widetilde{a} \mathrm{e}^{\Psi^{\mathfrak{g}}}, \widetilde{\rho} \mathrm{e}^{-\Psi^{\mathfrak{g}}}, 0\right)$. As the points of discontinuity of $\widetilde{a} \mathrm{e}^{\Psi}$ and $\widetilde{\rho} \mathrm{e}^{-\Psi}$ are included in those of $\widetilde{a} \mathrm{e}^{\Psi^{\mathfrak{g}}}$ and $\widetilde{\rho} \mathrm{e}^{-\Psi^{\mathfrak{g}}}$, it can be shown (see Prop. 6.2 in [7] for instance), that there is a constant $\tilde{C}$ depending on $T, \lambda, \Lambda, G,\|f\|_{\infty}$ and $\|\mathrm{d} f / \mathrm{d} x\|_{2}$, such that

$$
\sup _{(t, x) \in[0, T] \times G}\left|\mathbb{E}^{x} f\left(\widetilde{X}_{t}\right)-\mathbb{E}^{x} f\left(X_{t}^{\mathfrak{g}}\right)\right| \leq \tilde{C}\left(\left\|\widetilde{a} \mathrm{e}^{\Psi}-\widetilde{a} \mathrm{e}^{\Psi^{\mathfrak{g}}}\right\|_{\infty}^{2}+\left\|\widetilde{\rho} \mathrm{e}^{-\Psi}-\widetilde{\rho} \mathrm{e}^{-\Psi^{\mathfrak{g}}}\right\|_{\infty}\right)^{1 / 2}
$$

A simple application of the mean value theorem on each cell of the grid then leads to the desired result.

Proposition 7.1 means that it is easy to apply our scheme to $X^{\mathfrak{g}}$ because we have for this process explicit expressions of the quantities involved in step 2 of the algorithm.

Proposition 7.2 means that the weak error we make by approximating $\widetilde{X}$ by $X^{\mathfrak{g}}$ is the same as the approximation error of the scheme.

We used this approach in the numerical experiments presented in the next subsection.

\subsection{Numerical experiments}

We first test the efficiency of the scheme on some simple examples. We finally try to numerically estimate the speed of weak convergence of the scheme, on an elliptic PDE problem with inhomogeneous Dirichlet boundary conditions. 

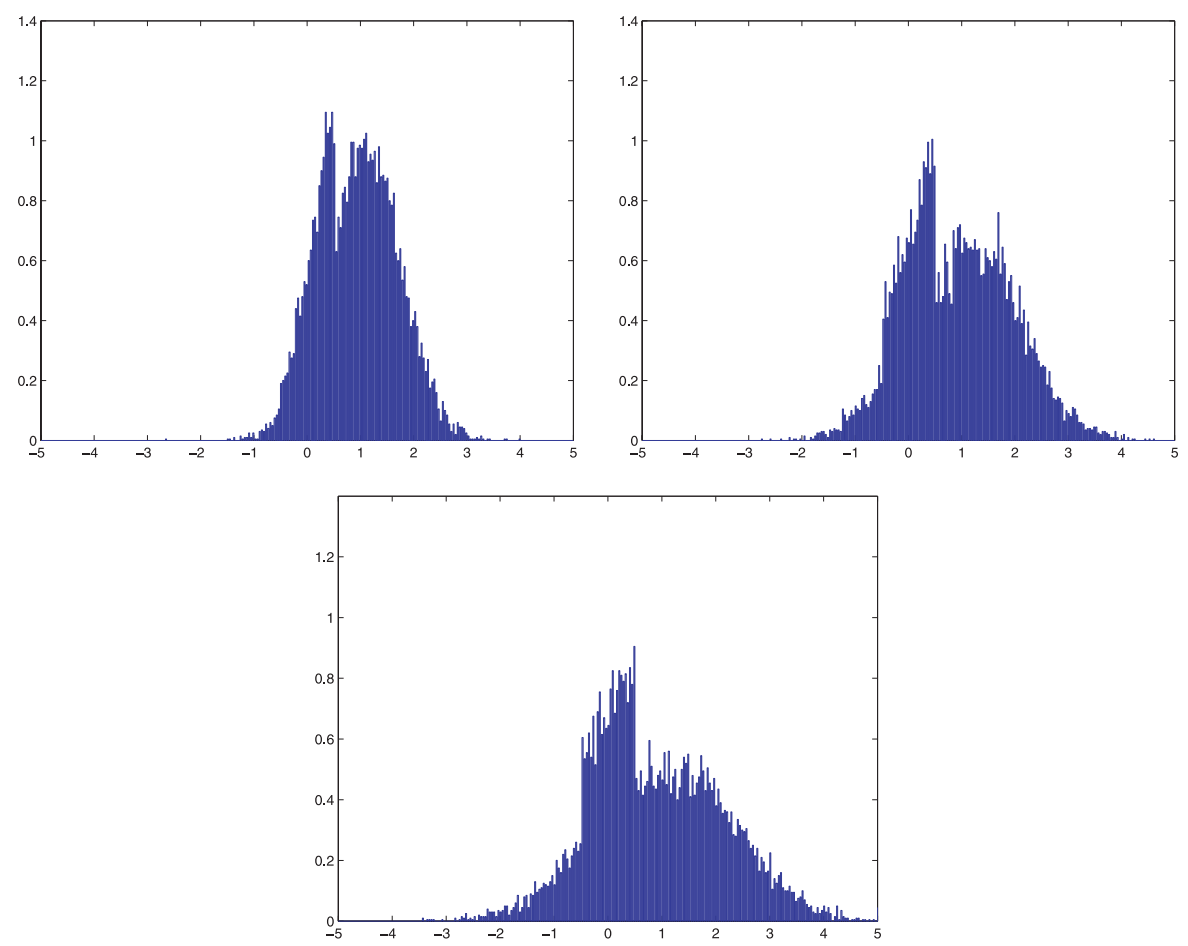

Figure 2. Approximation of $p(t, x, y)$ for $x=1.0$ at times $t=0.5, t=1.0$ and $t=1.5$ (with $N=10.000$ particles).

Example 7.3. We take $b=0$ and $a$ and $\rho$ to be

$$
a(x)=\left\{\begin{array}{ll}
1 & \text { if } x<-1 / 2 \\
2 & \text { if } x \in[-1 / 2,1 / 2) \\
1 & \text { if } x \geq 1 / 2,
\end{array} \text { and } \rho(x)= \begin{cases}1 & \text { if } x<-1 / 2 \\
1 / 2 & \text { if } x \in[-1 / 2,1 / 2) \\
1 & \text { if } x \geq 1 / 2\end{cases}\right.
$$

We take a domain $G=[-5,5]$ and a grid $\mathfrak{g}=\{k 0.02 \in G \mid k \in \mathbb{Z}\}$. We consider the process $X$ generated by $\mathfrak{L}(a, \rho, 0)$. As $a \rho=1$ everywhere, the average time spent by $X$ on a cell of $\mathfrak{g}$, knowing it has gone right or left, is the same as for the standard Brownian motion, that is to say $0.02^{2}$.

The jumps of $a$ in $-1 / 2$ and $1 / 2$ make that, heuristically, the process has a probability $2 / 3$ to go on the right in $-1 / 2$, while in $1 / 2$ this probability is $1 / 3$. Where the coefficient $a$ is flat, the process $X$ behaves like the Brownian motion and has a probability $1 / 2$ to go on the right. More precisely, we could describe the behavior of $X$ in terms of SDELT. Indeed $X$ solves

$$
X_{t}=X_{0}+W_{t}+\frac{1}{3} L_{t}^{-1 / 2}(X)-\frac{1}{3} L_{t}^{1 / 2}(X),
$$

where $L_{t}^{x}(X)$ is the symmetric local time of $X$ in $x$ (see [15]). We see that $X$ behaves like the Brownian motion $W$ except in $-1 / 2$ and $1 / 2$. Such a process $X$ is called Doubly Skew Brownian motion.

We simulate 10.000 paths of $\widehat{X}^{\mathfrak{g}}$ starting from $x=1.0$ at times $t=0.5, t=1.0$ and $t=1.5$. Compared to a Gaussian density centered in $x=1.0$ the histograms we get show a concentration of particles in the interval [-1/2,1/2] (Fig. 2).

Example 7.4. We take $G=[-5,5]$ and the grid $\mathfrak{g}=\{k 0.05 \in G \mid k \in \mathbb{Z}\}$. We simulate 10.000 paths of $\widehat{X}^{g}$ starting from $x=0.0$ and plot an histogram of the positions at time $t=1.0$. In Figure $2, X$ is generated by 


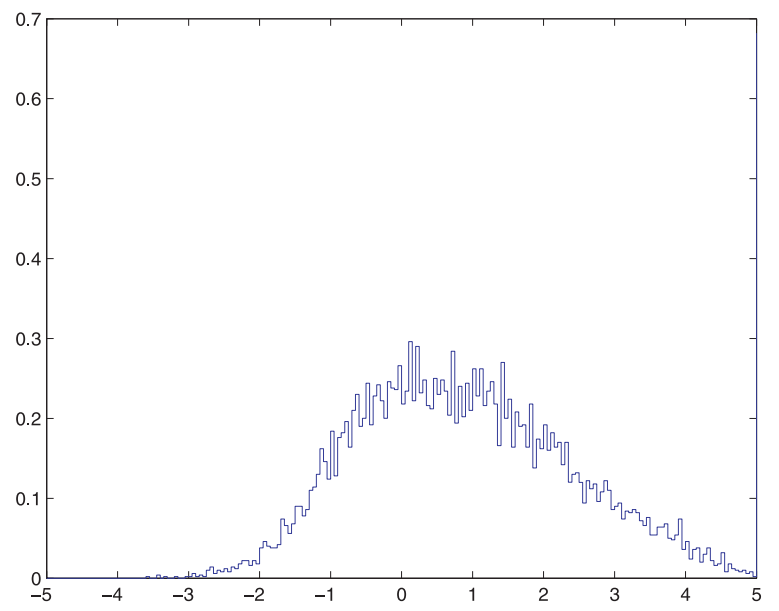

Figure 3. Histogram of the positions at time $t=1.0$ of 10.000 paths starting from $x=0.0$ of $X$ with $\rho=1$ and $a$ with value 1 on $\mathbb{R}^{+}$and 5 on $\mathbb{R}^{+}$.

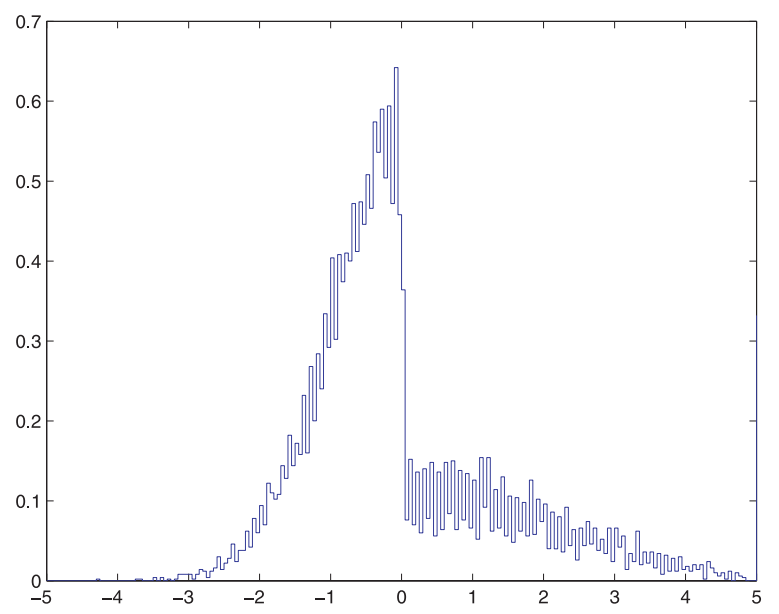

Figure 4 . Histogram of the positions at time $t=1.0$ of 10.000 paths starting from $x=0.0$ of $X$ with $a=1$ and $\rho$ with value 1 on $\mathbb{R}^{-}$and 5 on $\mathbb{R}^{+}$.

$\mathfrak{L}(a, \rho, 0)$ with $\rho=1$ and

$$
a(x)= \begin{cases}1 & \text { if } x<0 \\ 5 & \text { if } x \geq 0\end{cases}
$$

In Figure 3, $a=1$ and

$$
\rho(x)= \begin{cases}1 & \text { if } x<0 \\ 5 & \text { if } x \geq 0\end{cases}
$$

A comparison between Figures 3 and 4 shows that the process is more likely to go where $a$ is the highest and $\rho$ the lowest.

Example 7.5. We take $\rho=1, b=2$ and an irregular $a$ whose graph is plotted in Figure 5 . We know that $\mathbb{E}^{x} f\left(X_{t}\right)$ is the solution $u(t, x)$ of the parabolic problem $\partial u / \partial t=L u$, with initial condition $f$ and $(L, D(L))=$ $\mathfrak{L}(a, \rho, b)$. So the quantity $\frac{1}{N} \sum_{n=1}^{N} f\left(\widehat{X}_{t}^{\mathfrak{g}, n}\right)$ should approach $u(t, x)$. This is called a Monte Carlo method. 


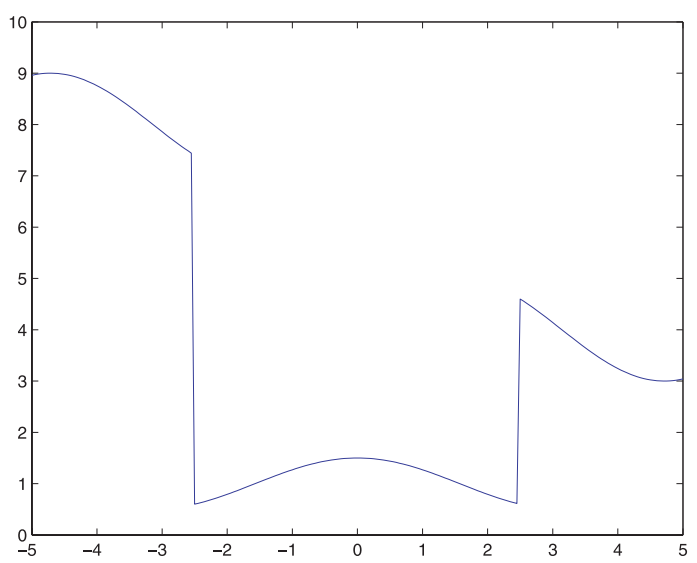

FiguRE 5. Graph of the coefficient $a$.
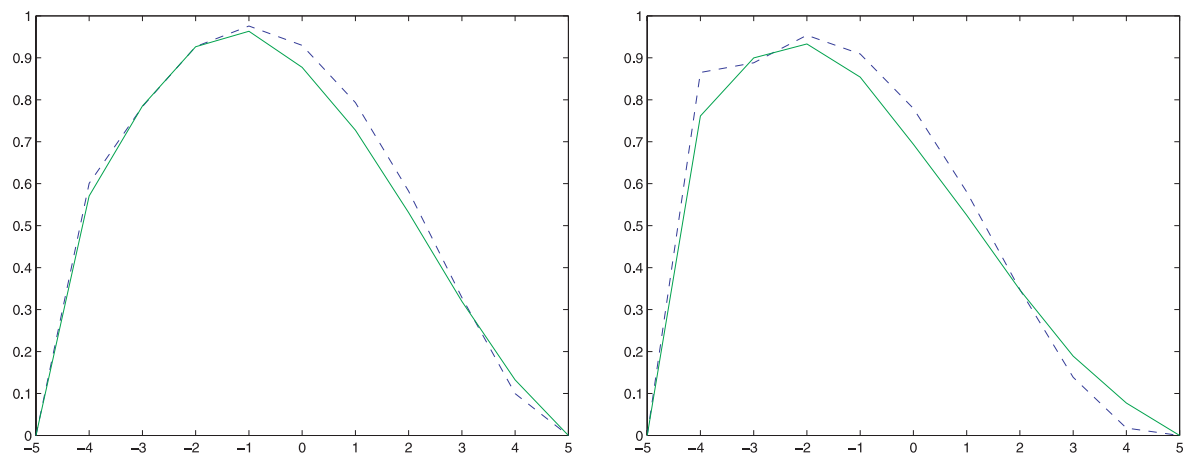

Figure 6. Graph of $\tilde{u}(t, \cdot)$ together with the one of $\bar{u}(t, \cdot)$ (represented by the dashed line), for $t=0.5$ and $t=1.0$.

We use the routine pdepe of MATLAB to compute with a deterministic method an approximation $\bar{u}(t, x)$ of $u(t, x)$, at times $t=0.5$ and $t=1.0$, with the initial condition

$$
f(x)=\cos \left(\frac{\pi}{10} x\right)
$$

Note that we have imposed uniform Dirichlet boundary conditions to the parabolic problem.

At each time $t \in\{0.5,1.0\}$ we simulate $N=8000$ paths of $\widehat{X}^{\mathfrak{g}}$ starting from $x \in\{-4,-3, \ldots, 3,4\}$ and compute for each $x$ the quantity

$$
\tilde{u}(t, x)=\frac{1}{N} \sum_{n=1}^{N} f\left(\widehat{X}_{t}^{\mathfrak{g}, n}\right) .
$$

We then plot on the same figure the graphs of $\bar{u}(t, \cdot)$ and $\tilde{u}(t, \cdot)$. Figure 6 shows a good adequation between the probabilistic and the deterministic approximations of $u(t, x)$.

Example 7.6 (numerical estimation of the weak error of the scheme on an elliptic problem). We now look at problems of the type

$$
\mathcal{P}(\alpha, \beta)\left\{\begin{array}{l}
L u=0 \text { on }(\ell, r) \\
u(\ell)=\alpha \\
u(r)=\beta
\end{array}\right.
$$



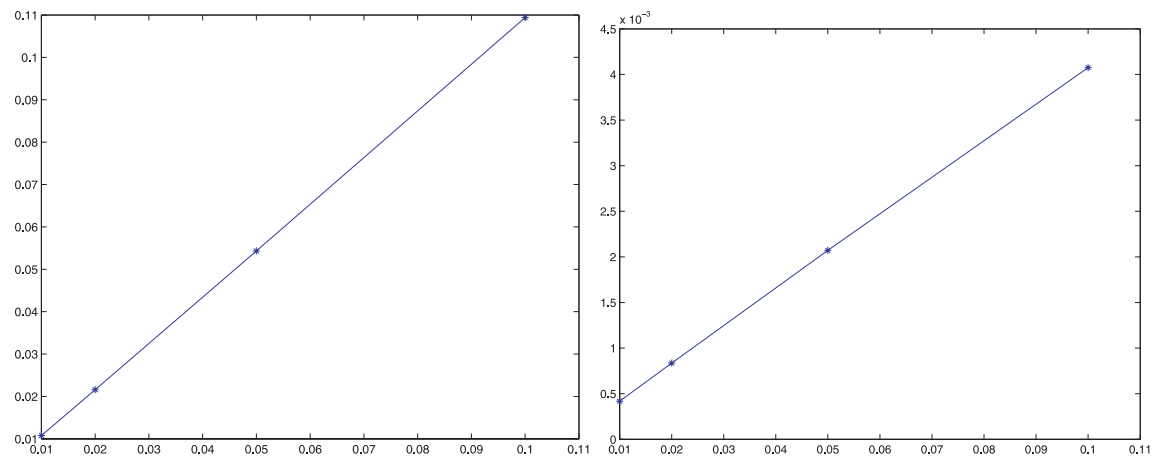

Figure 7 . Error between $\hat{u}(-3)$ and $u(-3)$ in function of the discretization step $h$.

Let us note $\psi(x)=\alpha \mathbb{1}_{\{x=\ell\}}+\beta \mathbb{1}_{\{x=r\}}$. By the Feynman-Kac formula we have for all $x \in(\ell, r), \mathbb{E}^{x}\left[\psi\left(X_{\tau}\right)\right]=$ $u(x)$, where $u$ is the solution of $\mathcal{P}(\alpha, \beta), X$ the process generated by $(L, D(L))$ and $\tau=\inf \left\{t \geq 0 \mid X_{t} \in\{\ell, r\}\right\}$.

Let us note $\widehat{S}^{\mathfrak{g}}$ the Markov chain on the grid $\mathfrak{g}$ defined by the probabilities $\pi(i, i+1)$ computed in step 2 of the algorithm. For $x \in G$ let us denote by $x_{j}(x)$ the point of $\mathfrak{g}$ that is the closest to $x$. Let us note $n^{*}=\inf \left\{p \in \mathbb{N} \mid \widehat{S}_{p}^{\mathfrak{g}} \in\{\ell, r\}\right\}$. An approximation of $u(x)$ is given by

$$
\hat{u}(x)=\mathbb{E}^{x_{j}(x)}\left[\psi\left(\widehat{S}_{n^{*}}^{\mathfrak{g}}\right)\right] .
$$

Indeed $\widehat{S}_{n^{*}}^{\mathfrak{g}}$ has the same law as $\widehat{X}_{\hat{\tau}}^{\mathfrak{g}}$, where $\hat{\tau}=\inf \left\{t \geq 0 \mid \widehat{X}_{t}^{\mathfrak{g}} \in\{\ell, r\}\right\}$. By the strong Markov property we get, for $x=x_{j}$,

$$
\hat{u}\left(x_{j}\right)=\mathbb{E}^{x_{j}}\left[\mathbb{E}\left[\psi\left(\widehat{S}_{n^{*}}^{\mathfrak{g}}\right) \mid \widehat{S}_{1}^{\mathfrak{g}}\right]\right]=\mathbb{E}^{x_{j}}\left[\hat{u}\left(S_{1}\right)\right]=\hat{u}\left(x_{j+1}\right) \pi(j, j+1)+\hat{u}\left(x_{j-1}\right) \pi(j, j-1) .
$$

Consider the grid $\mathfrak{g}=\left\{x_{j}\right\}_{j=0, \ldots, J}$ on $(\ell, r)$ with $l=x_{0}$ and $r=x_{J}$. Define the vectors $U$ and $F$ by $U=\left(\hat{u}\left(x_{1}\right), \ldots, \hat{u}\left(x_{J-1}\right)\right)^{\top}$ and $F=(\alpha \pi(1,0), 0, \ldots, 0, \beta \pi(J-1, J))^{\top}$. Let $\Pi$ be the matrix whose non-zero coefficients are the $\Pi(j, j \pm 1)=\pi(j, j \pm 1)$. The vector $U$ is solution of the linear system

$$
(I-\Pi) U=F .
$$

Thus the quantity $\mathbb{E}^{x_{j}(x)}\left[\psi\left(\widehat{S}_{n^{*}}^{\mathfrak{g}}\right)\right]=\hat{u}(x)$ can be computed without using any Monte Carlo method. We can thus get rid of any statistical error: the numerical error will be entirely due to the discretization error between $\mathbb{E}^{x_{j}(x)}\left[\psi\left(\widehat{S}_{n^{*}}^{\mathfrak{g}}\right)\right]=\mathbb{E}^{x}\left[\psi\left(\widehat{X}_{\hat{\tau}}^{\mathfrak{g}}\right)\right]$ and $\mathbb{E}^{x}\left[\psi\left(X_{\tau}\right)\right]$.

We take $l=-5, r=5, \alpha=3$ and $\beta=1$. We take $\rho=1$ but actually in a problem of type $\mathcal{P}(\alpha, \beta)$ the solution is the same whatever the value of $\rho$. We first take $b=2$ and the same $a$ as in Example 3. We second take $b=0$ and

$$
a(x)= \begin{cases}2+\sin (x) & \text { if } x<0 \\ 5+\sin (x+\pi) & \text { if } x \geq 0 .\end{cases}
$$

In each case we take $\mathfrak{g}=\{h k \mid k \in \mathbb{Z}\}$ with $h$ taking the successive values $h=0.1,0.05,0.02,0.01$. We evaluate the error between $\hat{u}(x)$ and some accurate deterministic approximation of $u(x)$ at the point $x=-3$. The graphs we obtain are shown in Figure 7.

These graphs show a linear growth of the weak error. In the case of an Euler scheme for smooth coefficients, it is a classical fact to have for instance a strong error of order $\mathcal{O}(\sqrt{\Delta t})$ and a weak error of order $\mathcal{O}(\Delta t)$ (see for example the book [12]). But the mechanism of our scheme is different from the one of an Euler scheme and no regularity is assumed. We were not able to give a theoretical reason for such a convergence for our numerical scheme, that shows a weak error of order $\mathcal{O}(h)$ while the strong error is rather of order $\mathcal{O}(\sqrt{h})$. 


\section{REFERENCES}

[1] A. Bensoussan, J.L. Lions and G. Papanicolaou, Asymptotic Analysis for Periodic Structures. North-Holland, Amsterdam (1978).

[2] P. Billingsley, Convergence of Probabilities Measures. John Wiley \& Sons (1968).

[3] L. Breiman, Probability. Addison-Wesley Series in Statistics (1968).

[4] H. Brezis, Analyse fonctionnelle. Masson (1983).

[5] M. Decamps, A. De Schepper and M. Goovaerts, Applications of $\delta$-function pertubation to the pricing of derivative securities. Physica A 342 (2004) 677-692.

[6] M. Decamps, M. Goovaerts and W. Schoutens, Self Exciting Threshold Interest Rates Model. Int. J. Theor. Appl. Finance 9 (2006) 1093-1122.

[7] P. Étoré, On random walk simulation of one-dimensional diffusion processes with discontinuous coefficients. Electron. J. Probab. 11 (2006) 249-275.

[8] P. Étoré, Approximation de processus de diffusion à coefficients discontinus en dimension un et applications à la simulation. Ph.D. thesis, Université Henri Poincaré, Nancy, France (2006).

[9] O. Faugeras, F. Clément, R. Deriche, R. Keriven, T. Papadopoulo, J. Roberts, T. Viéville, F. Devernay, J. Gomes, G. Hermosillo, P. Kornprobst and D. Lingrand, The inverse EEG and MEG problems: The adjoint state approach I: The continuous case. INRIA research report RR-3673 (1999).

[10] M. Freidlin and A.D. Wentzell, Necessary and Sufficient Conditions for Weak Convergence of One-Dimensional Markov Processes. Festschrift dedicated to 70th Birthday of Professor E.B. Dynkin, Birkhäuser (1994) 95-109.

[11] V.V. Jikov, S.M. Kozlov and O.A. Oleinik, Homogenization of Differential Operators and Integral Functionals. Springer (1994).

[12] P.E. Kloeden and E. Platen, Numerical Solution of Stochastic Differential Equations. Springer, Berlin (1992).

[13] A. Lejay, Méthodes probabilistes pour l'homogénéisation des opérateurs sous forme divergence: cas linéaires et semi-linéaires. Ph.D. thesis, Université de Provence, Marseille, France (2000).

[14] A. Lejay, Stochastic Differential Equations Driven by Processes Generated by Divergence Form Operators I: A Wong-Zakai Theorem. ESAIM Probab. Stat. 10 (2006) 356-379.

[15] A. Lejay and M. Martinez, A scheme for simulating one-dimensional diffusion processes with discontinuous coefficients. Annals Appl. Probab. 16 (2006) 107-139.

[16] M. Martinez, Interprétations probabilistes d'opérateurs sous forme divergence et analyse de méthodes numériques probabilistes associées. Ph.D. thesis, Université de Provence, Marseille, France (2004).

[17] M. Martinez and D. Talay, Discrétisation d'équations différentielles stochastiques unidimensionnelles à générateur sous forme divergence avec coefficient discontinu. C.R. Acad. Sci. Paris 342 (2006) 51-56.

[18] H. Owhadi and L. Zhang, Metric based upscaling. Commun. Pure Appl. Math. (to appear).

[19] J.M. Ramirez, E.A. Thomann, E.C. Waymire, R. Haggerty and B. Wood, A generalized Taylor-Aris formula and Skew Diffusion. Multiscale Model. Simul. 5 (2006) 786-801.

[20] D. Revuz and M. Yor, Continuous Martingale and Brownian Motion. Springer, Heidelberg (1991).

[21] A. Rozkosz, Weak convergence of Diffusions Corresponding to Divergence Form Operators. Stochastics Stochastics Rep. $\mathbf{5 7}$ (1996) 129-157.

[22] D.W. Stroock, Diffusion semigroups corresponding to uniformly elliptic divergence form operators. Springer, Lecture Notes in Mathematics, Seminaire de Probabilités XXII 1321 (1988) 316-347.

[23] D.W. Stroock and W. Zheng, Markov chain approximations to symmetric diffusions. Ann. Inst. H. Poincaré Probab. Statist. 33 (1997) 619-649.

[24] V.V. Zhikov, S.M. Kozlov, O.A. Oleinik and K. T'en Ngoan, Averaging and G-convergence of Differential Operators. Russian Math. Survey 34 (1979) 69-147.

[25] V.V. Zhikov, S.M. Kozlov and O.A. Oleinik, G-convergence of Parabolic Operators. Russian Math. Survey 36 (1981) 9-60. 